\title{
MicroRNA-148a facilitates inflammatory dendritic cell differentiation and autoimmunity by targeting MAFB
}

\author{
Yao Meng, ${ }^{1}$ Jun Li, ${ }^{1}$ Zhizhong Ye, ${ }^{2}$ Zhihua Yin, ${ }^{2}$ Qing Sun, ${ }^{1}$ Zhuojun Liao, ${ }^{1}$ Guanhua Li, ${ }^{1}$ Jun Deng, \\ Lu Liu, ${ }^{3}$ Yuqing Yu, ${ }^{1} \mathrm{Li} \mathrm{Wu},{ }^{4}$ Haibo Zhou, ${ }^{1,2}$ and Nan Shen ${ }^{1,2,5,6,7}$ \\ ${ }^{1}$ Shanghai Institute of Rheumatology, Renji Hospital, Shanghai Jiao Tong University School of Medicine (SJTUSM), \\ Shanghai, China. ${ }^{2}$ Shenzhen Futian Hospital for Rheumatic Diseases, Shenzhen, China. ${ }^{3}$ Institute of Dermatology and \\ Department of Dermatology, First Affiliated Hospital, Anhui Medical University, Hefei, Anhui, China. ${ }^{4}$ Tsinghua-Peking \\ Joint Center for Life Sciences, Tsinghua University School of Medicine, Beijing, China. ${ }^{5}$ Center for Autoimmune Genomics \\ and Etiology, Cincinnati Children's Hospital Medical Center, Cincinnati, Ohio, USA. ${ }^{6}$ Department of Pediatrics, University of \\ Cincinnati College of Medicine, Cincinnati, Ohio, USA. 'State Key Laboratory of Oncogenes and Related Genes, Shanghai \\ Cancer Institute, Renji Hospital, SJTUSM, Shanghai, China.
}

\begin{abstract}
Monocyte-derived DCs (moDCs) have been implicated in the pathogenesis of autoimmunity, but the molecular pathways determining the differentiation potential of these cells remain unclear. Here, we report that microRNA-148a (miR-148a) serves as a critical regulator for moDC differentiation. First, miR-148a deficiency impaired the moDC development in vitro and in vivo. A mechanism study showed that MAFB, a transcription factor that hampers moDC differentiation, was a direct target of $m i R-148 a$. In addition, a promoter study identified that $m i R-148 a$ could be transcriptionally induced by PU.1, which is crucial for moDC generation. miR-148 a ablation eliminated the inhibition of PU.1 on MAFB. Furthermore, we found that miR-148a increased in monocytes from patients with psoriasis, and miR-148a deficiency or intradermal injection of antagomir-148a immensely alleviated the development of psoriasis-like symptoms in a psoriasis-like mouse model. Therefore, these results identify a pivotal role for the PU.1-miR-148a-MAFB circuit in moDC differentiation and suggest a potential therapeutic avenue for autoimmunity.
\end{abstract}

Authorship note: YM and JL contributed equally to this work.

Conflict of interest: The authors have declared that no conflict of interest exists.

Copyright: () 2020, American Society for Clinical Investigation.

Submitted: September 23, 2019

Accepted: March 18, 2020

Published: April 23, 2020

Reference information: /CI Insight. 2020;5(8):e133721.

https://doi.org/10.1172/jici.

insight.133721.

\section{Introduction}

DCs comprise a heterogeneous family of professional antigen-presenting cells that maintain the homeostasis of the immune system (1-4). DC populations in the skin have been well investigated in mice (5-7). In the steady state, two major subsets of conventional DCs (cDCs) have been characterized in the mouse nonlymphoid tissues, $\mathrm{CD} 103^{+} \mathrm{CD} 11 \mathrm{~b}^{-} \mathrm{cDCs}$ and $\mathrm{CD} 103^{-} \mathrm{CD} 11 \mathrm{~b}^{+} \mathrm{cDCs}$, whereas the epidermis contains Langerhans cells. In the inflammatory state, peripheral monocytes are rapidly recruited to inflammatory sites and give rise to monocyte-derived DCs (moDCs) (5-7). Moreover, an increased frequency and activation status of plasmacytoid DCs (pDCs), a potent type I IFN-producing DC subset, have been documented in early psoriatic lesions $(8,9)$. Both $\mathrm{cDCs}$ and $\mathrm{pDCs}$ originate from DC precursors in a Fms-related tyrosine kinase 3 ligand (Flt3L)-dependent manner, whereas the generation of moDCs is GM-CSF dependent $(1,2)$.

$\mathrm{Up}$ to now, the regulation of moDC activation in autoimmune diseases has been studied extensively (10-13). Studies have demonstrated that transcriptional factors, such as STAT5, PU.1, and MAFB, affect moDC generation at different stages of hematopoietic differentiation (14-20). Both PU.1 and MAFB are involved in the myeloid commitment of hematopoietic stem cells, and the balance between them specifies alternative DC or macrophage fate $(18,19)$. Despite intensive study, the molecular mechanism underlying regulatory network for moDC generation is far from clarified.

Psoriasis is a common, chronic autoinflammatory skin disease characterized by demarcated, red, and scaly plaques. Environmental and genetic factors trigger the hyperproliferation and disturbed differentiation of keratinocytes, thickening of the epidermis, formation of new blood vessels, and accumulation of leukocytes in epidermis and dermis, of which T cells and DCs are the most critical $(21,22)$. Psoriatic skin contains large numbers of activated DCs, which produce IL-23, IL-12, IL-6, and TNF- $\alpha$. 
They activate and polarize autoaggressive Th cells toward Th17, Th1, and Th22 cell subsets defined by the production of IL-17A, IL-17F, IFN- $\gamma$, and IL-22, respectively $(9,23,24)$. Recently, the involvement of different skin DC subsets in regulating psoriatic plaque formation has been studied extensively. pDCs and $\mathrm{CDCs}$ are dispensable for the local skin inflammation in psoriasis, since $E 2-2^{-/}$mice and Flt3L $\mathrm{L}^{-/-}$ mice presented no significant difference in ear swelling and epidermal thickening compared with WT mice $(25,26)$. The $C c r 2^{-1-}$ mice, which have profound monocytopenia due to a defect in monocyte egress from BM, showed a significant reduction in skin pathological phenotype (26). Depletion of monocytes in vivo through the use of antibody also greatly relieved the symptoms (26). The function of Langerhans cells is controversial because Lan-DTR mice developed a degree and course of psoriasiform skin disease similar to those of WT mice in an imiquimod-induced (IMQ-induced) model (25) but showed a certain degree of improvement in an IL-23-induced model (26). The finding that locally increased expression of GM-CSF and IL-23 was exclusively produced by Langerin- DCs in vivo, which further supports moDCs as being the most important DC subset in psoriasis (25).

miRNAs are short ( $\sim 22 \mathrm{nt})$, evolutionarily conserved, single-stranded RNAs that control the expression of complementary target mRNAs, leading to their transcript destabilization, translational inhibition, or both (27). miRNAs are critical regulators of immune cell development and function (28). In this study, we used the psoriasis disease model to identify $m i R-148 a$ as a paramount regulator for autoimmune-related moDC differentiation. miR-148a deficiency led to decreased pathogenic moDCs. Consequently, either $m i R-148$ a knockout or $m i R-148 a$ inhibition by intradermal administration with antagomir-148a prevented the development of moDCs and psoriasis-like inflammation in the IMQ-induced psoriasis-like mouse model. The mechanism research revealed that $M A F B$ was a bona fide direct target of $m i R-148 a$, which was transcriptionally regulated by PU.1. Therefore, these results identify a pivotal role for the PU.1-miR-148a-MAFB pathway in the differentiation of moDCs and suggest a potential therapeutic avenue for inflammatory moDCs mediated autoimmune diseases.

\section{Results}

miR-148a is indispensable for moDC differentiation. Proinflammatory moDC infiltration is critical for the progression of several autoimmune diseases, including systemic lupus erythematosus (SLE), psoriasis, inflammatory bowel disease, and so on $(25,29,30)$. A comprehensive analysis of our previous data in patients with SLE (31) or psoriasis revealed an increase in the expression of miR-148a-3p (referred to as $m i R-148 a$ here) (Supplemental Figure 1; supplemental material available online with this article; https://doi.org/10.1172/jci.insight.133721DS1). Other groups also reported similar elevation in autoimmune diseases (32). Therefore, we hypothesized that $m i R-148 a$ might affect the development or function of pathogenic moDCs in autoimmune diseases.

Before evaluating the function of $m i R-148 a$ in psoriatic inflammation, we first checked the effect on hematopoietic differentiation in the steady state (Supplemental Figure 2, A-D). Accordingly, different cell subsets in the spleen and skin from $m i R-148 a^{-/-}$and WT littermate mice were detected (Supplemental Figure 2, A and C). Consequently, there was no difference in the number of lymphoid or myeloid cells (Supplemental Figure 2, B and D), indicating that miR-148a was dispensable for hematopoietic differentiation in the steady state.

In the inflammatory state, $\mathrm{Ly} 6 \mathrm{C}^{\text {hi }}$ monocytes migrate to the lesion sites and then differentiate into moDCs. To validate whether the DC differentiation from monocytes was affected by $m i R-148 a$, Ly6C hi monocytes were isolated from BM of $m i R-148 a^{-1-}$ and WT mice and then cultured with GM-CSF and IL-4. It has been demonstrated that Ly6 $\mathrm{C}^{\mathrm{hi}}$ monocytes can give rise to both $\mathrm{CD} 11 \mathrm{c}^{+} \mathrm{MHCII} \mathrm{I}^{\mathrm{hi}} \mathrm{CD} 11 \mathrm{~b}^{\mathrm{int}} \mathrm{DC}$ and $\mathrm{CD} 11 \mathrm{c}^{+} \mathrm{MHCII}{ }^{\text {int }} \mathrm{CD} 11 \mathrm{~b}^{\text {hi }}$ macrophages (10). The conclusion was confirmed in the culture system that sorted CD $11 \mathrm{c}^{+} \mathrm{MHCII}{ }^{\text {hi }} \mathrm{CD} 11 \mathrm{~b}^{\text {int }}$ cells exhibited DC morphology and CD $11 \mathrm{c}^{+} \mathrm{MHCII}{ }^{\text {int }} \mathrm{CD} 11 \mathrm{~b}^{\text {hi }}$ cells had a typical macrophage morphology (Figure 1, A and B), allowing us to evaluate the differentiation potential of monocytes toward macrophages and DCs in the same culture system.

There was no difference of the expanding potential of monocytes between control and miR-148a

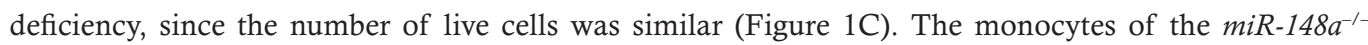
group had considerably less moDCs (about 70\% reduction) than those of controls (Figure 1, A and C). In contrast, both the percentage and the number of macrophages increased in the $m i R-148 a^{-/-}$group (Figure $1, \mathrm{~A}$ and $\mathrm{C}$ ), indicating a critical role for $m i R-148 a$ in promoting the differentiation of monocytes toward 


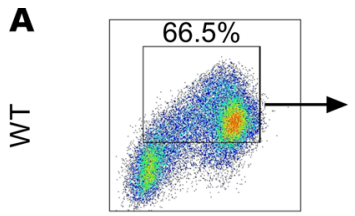

33.4\% Macrophage
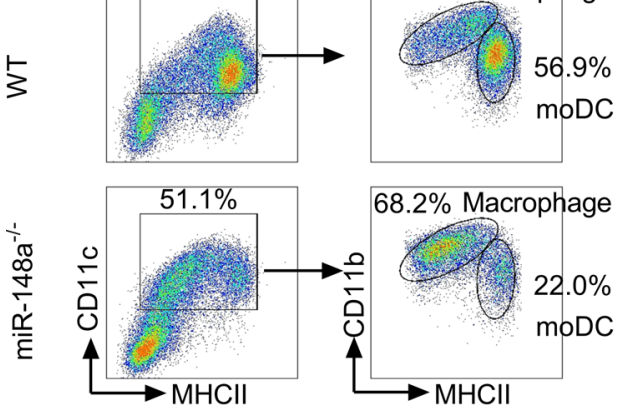

B
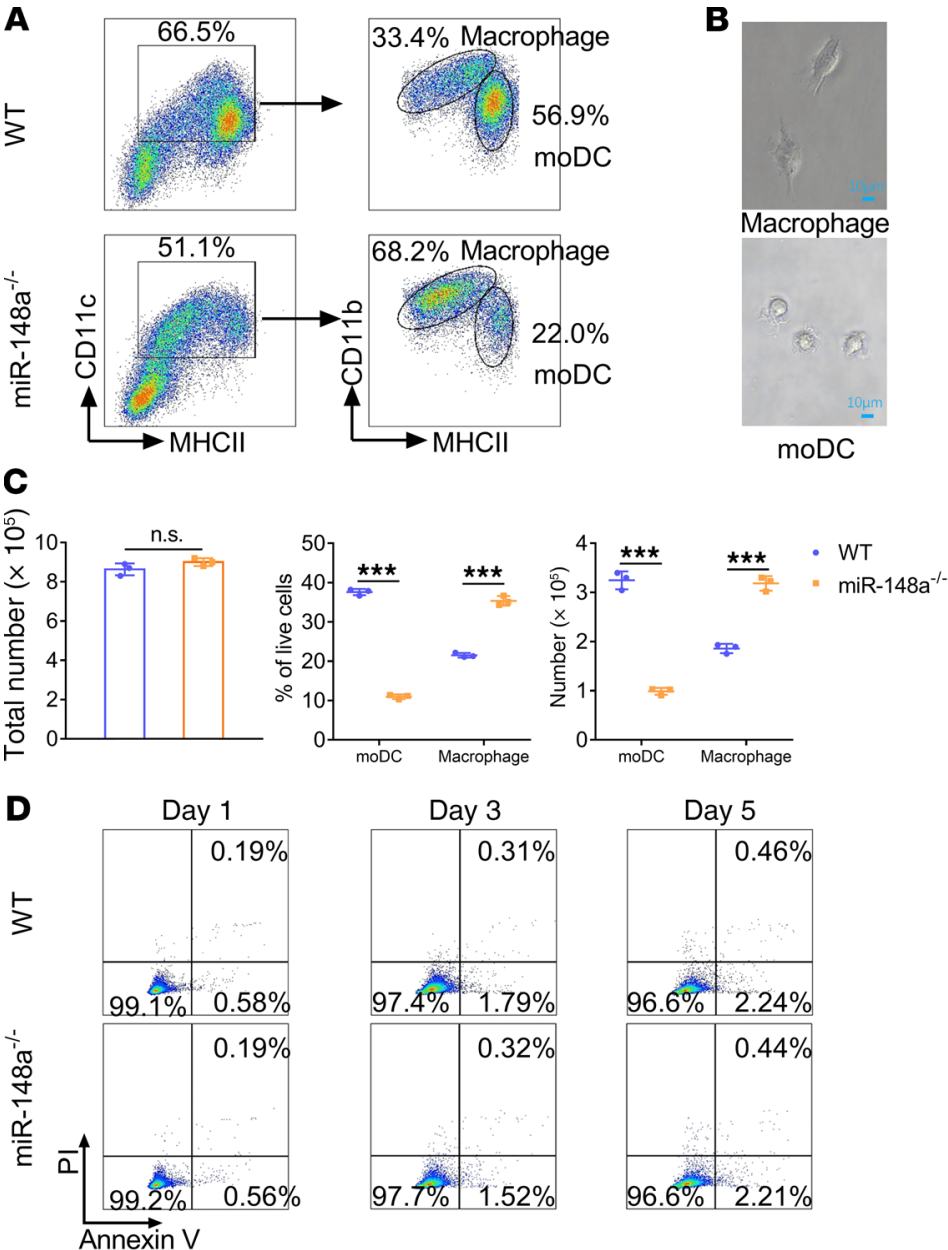

Day 3

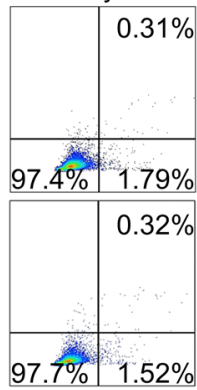

Day 5

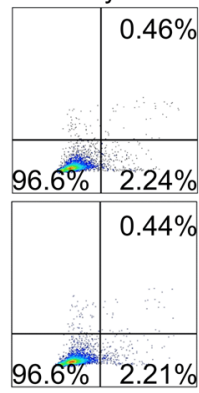

$96.6 \% \quad 2.21 \%$
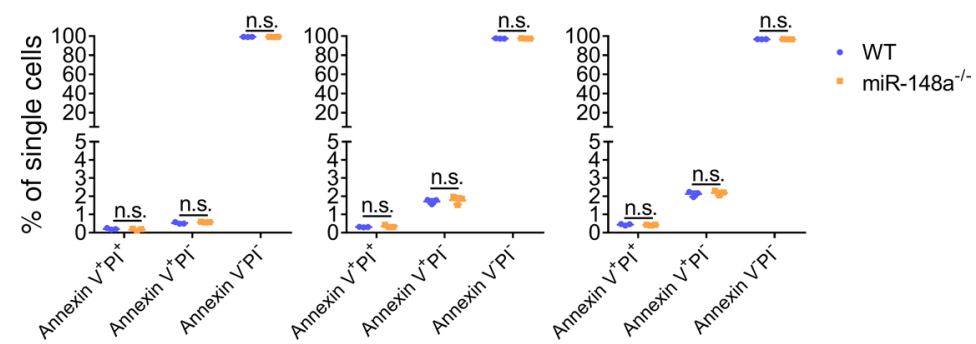

Figure 1. $m i R-148 a$ is indispensable for moDC differentiation. (A and B) Monocytes isolated from BM of WT or miR-148a 1 - mice were cultured with $50 \mathrm{ng} / \mathrm{mL}$ GM-CSF and $20 \mathrm{ng} / \mathrm{mL} \mathrm{IL-4}$ for 5 days. Flow cytometry analysis (A), morphology (B), and statistical data (C) of moDCs and macrophages are shown. (D) Flow cytometry and statistical data of apoptosis during monocyte differentiation at indicated time points were analyzed $(n=3)$. Scale bars: $10 \mu \mathrm{m}$ (B). $P$ values were determined by 2 -tailed unpaired $t$ test. Data are shown as mean $\pm \mathrm{SEM}$. ${ }^{* * *} P<0.001$, n.s., not significant). miR-148a, microRNA-148a; moDC, monocyte-derived DC.

inflammatory DCs at the expense of macrophage.

Previous studies reported that $m i R-148 a$ was involved in antiapoptosis and frequently upregulated in primary and tumor cells $(33,34)$. To confirm whether the decrease in the proportion and number of miR-148a-1moDCs was a result of the increased apoptosis, the entire cells were stained with annexin $\mathrm{V}$ and propidium iodide. Consequently, the examination of cell apoptosis showed no difference between miR-148$a^{-1-}$ and WT throughout the inducing process, suggesting that miR-148a did not affect the moDC survival (Figure 1D).

$M A F B$ is a direct target of miR-148a. To gain insight into the molecular mechanism and thus facilitate the differentiation of moDCs for miR-148a, we performed RNA-sequencing analysis of monocytes iso-

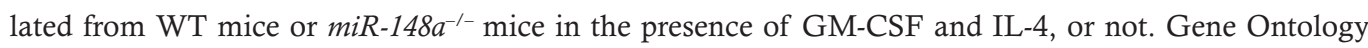
(GO) analysis showed that autoimmune disease-related pathways were enriched with obviously changed 
genes (SLE, RA, etc.), suggesting that miR-148a was important in autoimmune response (Figure 2A). Since miRNA promotes the degradation of its target gene mRNAs, the mRNA abundance of $m i R-148 a$ target gene should be higher in $m i R-148 a^{-/-}$monocytes than in WT monocytes. The threshold was set at $\pm 0.2 \mathrm{CT}$, since the degrading potential of target mRNA by miRNA is much weaker than siRNA. Then, we identified 990 protein-coding genes upregulated in their mRNA abundance in $m i R-148 a^{-/-}$monocytes

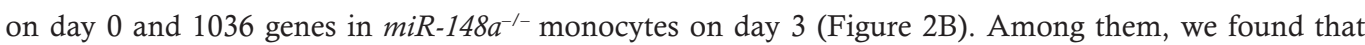
136 genes were upregulated on both day 0 and day 3 in $m i R-148 a^{-1-}$ monocytes. Finally, bioinformatic analysis helped confirm that 7 of 136 genes contained predicted conserved miR-148a-binding sites (Figure 2B). Targets previously implicated in hematopoietic differentiation were of special interest because the results suggested a role for $m i R-148 a$ in regulating moDC generation. Of these 7 genes, MAFB has been reported to be increased during the differentiation from monocytes to macrophages and decreased during the differentiation from monocytes to moDCs $(18,20)$. Overexpression of MAFB in myeloid progenitor cells is proposed to induce macrophage differentiation at the expense of moDC differentiation (19). These resulted in the selection of MAFB for further study (Figure 2C).

The RNA-sequencing results were validated by qRT-PCR (Supplemental Figure 3A), and the protein expression encoded by $M A F B$ was examined by immunoblot (Figure 2D; see complete unedited blots in the supplemental material). Both the mRNA and protein levels of MAFB increased in $m i R-148 a^{-1-}$ monocytes. We transfected human primary monocytes with antagomir-148a as well. The results showed that knockdown of miR-148a led to the increase of MAFB expression (Supplemental Figure 3D; see complete unedited blots in the supplemental material).

To test the direct binding of miR-148a with $M A F B$ mRNA, we performed the RNA-binding protein immunoprecipitation (RIP) assay using an antibody against Argonaute-2 (Ago2), a component of the RNA-induced silencing complex that mediates miRNA-directed gene silencing. The results showed that overexpression of $m i R-148 a$ significantly enriched the association of $M A F B$ mRNA with the Ago2-containing complex, suggesting that the $M A F B$ mRNA was directly bound by $m i R-148 a$ (Figure $2 \mathrm{E}$ ). Furthermore, to confirm the effect of miR-148a on the $M A F B$ 3'UTR, dual-luciferase reporter assays were conducted. Gene fragments encoding 3'UTR region of $M A F B$ were cloned into the expression vector psiCHECK2, which encodes the renilla luciferase controlled by the cloned 3'UTR. These constructs were cotransfected in HEK 293T cells with miR-148a mimic. Data showed that miR-148a overexpression significantly reduced reporter protein expression through the 3 'UTR region of $M A F B$ (Figure $2 \mathrm{~F}$ ). When the predicted binding site for $m i R-148 a$ was mutated, the reduction was abolished (Figure $2 \mathrm{~F}$ ). Therefore, $m i R-148 a$ can suppress the expression of MAFB through its cognate binding site in 3'UTR.

Since miR-148a directly regulates $M A F B$ expression, it is crucial to confirm that the higher protein level

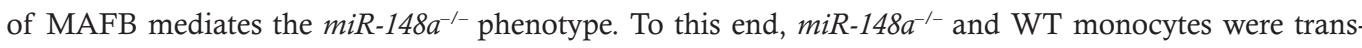
fected with specific siRNA for $M A F B$ and then cultured with GM-CSF and IL-4. Knockdown of MAFB promoted the moDC differentiation potential of $m i R-148 a^{-1-}$ monocytes such that it was comparable to that of the WT monocytes (Figure 2G and Supplemental Figure 3, B and C; see complete unedited blots in the supplemental material). These data have therefore demonstrated that MAFB mediated the phenotype of $m i R-148 a$ deficiency and confirmed that $M A F B$ was a bona fide direct target of miR-148a.

$m i R-148 a$ is involved in the inhibition of $M A F B$ expression by PU.1. Considering that miR-148a can regulate moDC differentiation, we then explored how this miRNA was transcriptionally regulated. Previous studies have identified the transcriptional start sites (TSSs) of miR-148a (35). Intriguingly, a conserved PU.1 binding sites was identified in the promoter (Figure 3A). To validate whether PU.1 can modulate miR-148a expression, we transfected primary monocytes with PU.1 siRNA. Knockdown of PU.1 led to the reduction of miR-148a expression (Figure 3B and Supplemental Figure 4, A and B; see complete unedited blots in the supplemental material). To further explore the physiological relevance of PU.1-dependent regulation of $m i R-148 a$ and subsequent MAFB expression, we measured the dynamic changes of PU.1, miR-148a, and MAFB during moDC differentiation. As expected, the miR-148a level paralleled the increase in PU.1 mRNA and protein levels, while the miR-148a level showed an opposite trend compared with the MAFB mRNA and protein levels (Figure 3, C and D, and Supplemental Figure 4C; see complete unedited blots in the supplemental material).

To further determine whether miR-148a participated in the inhibition of MAFB expression by PU.1, both $m i R-148 a^{-1-}$ and WT monocytes were transfected with PU.1 siRNA. The results manifested that the inhibitory effect of PU.1 on MAFB expression disappeared in the $m i R-148 a^{-1-}$ monocytes (Figure $3 \mathrm{E}$ and Supplemental Figure 4, D and E; see complete unedited blots in the supplemental material). Therefore, 
A

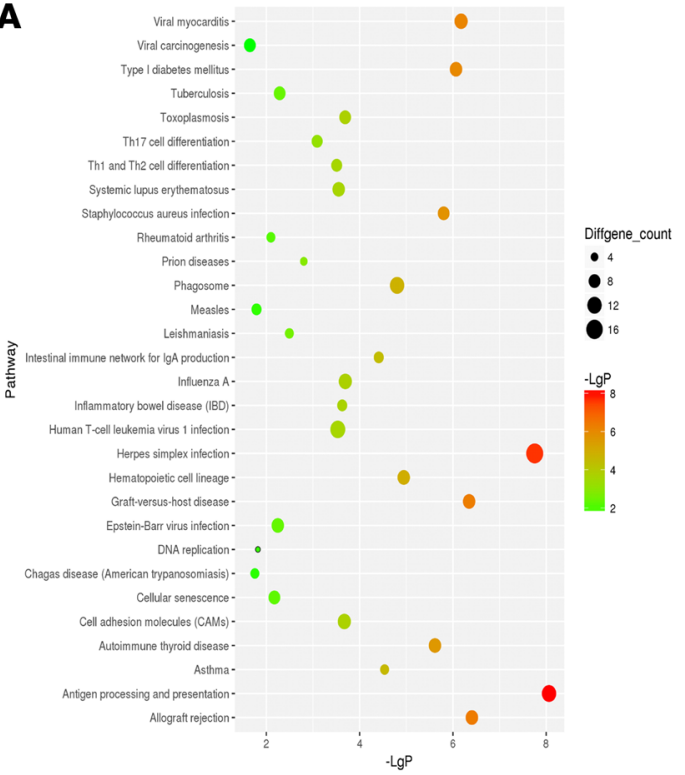

C

Mafb 3'UtR 5,...AguaAaAggauUuaAguUgcacuga... Human 5' $\cdots$ AGUAAGAGGAUUCG-GUUGCACUGA miR-148a $33^{\prime}$ UGUUUCAAGACAUC-ACGUGACU

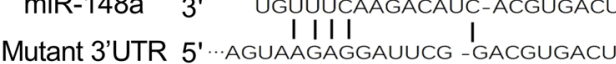
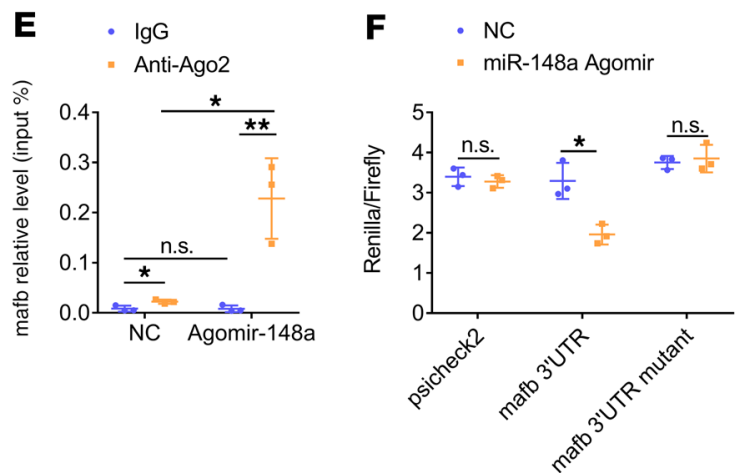

B

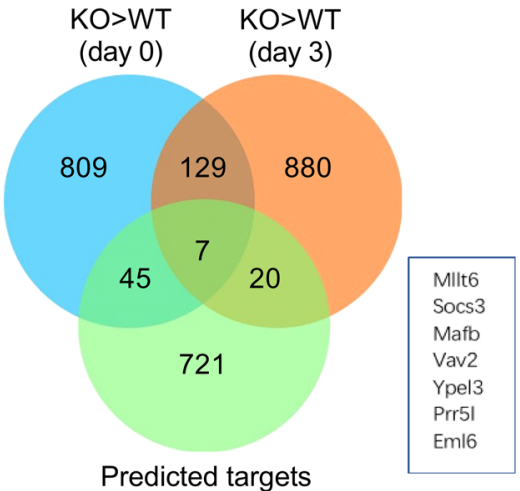

D
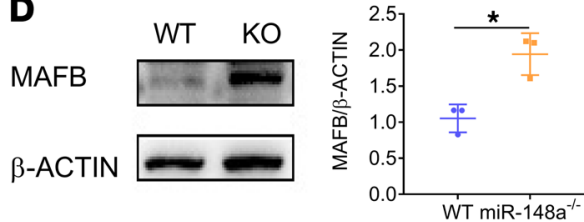

- $\mathrm{WT}+\mathrm{NC}$

- $\mathrm{miR}-148 \mathrm{a}^{-1-}+\mathrm{NC}$

C. WT+mafb siRNA

G $\quad$ miR-148a $a^{-1-}+m a f b ~ s i R N A$

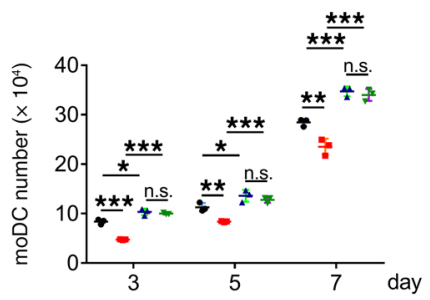

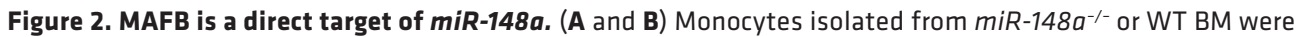
cultured with GM-CSF and IL-4, or not. Gene expression were detected by mRNA sequencing. (A) Gene Ontology analysis of differentially expressed genes. (B) Venn diagram of upregulated genes in miR-148a $a^{-/-}$monocytes on days 0 and 3 is shown. Upregulated genes with predicted miR-148a targets are listed $(n=3)$. (C) Predicted binding site of miR-148a in the 3'UTR of MAFB and the mutation of binding site. (D) Immunoblot analysis of MAFB expression in monocytes isolated from WT or $m i R-148 a^{-/-}$BM. The target gene protein/ $\beta$-ACTIN ratio in the WT group was set as 1. (E) RNA immunopurification assay. Human primary monocytes were transfected with control (NC) or Agomir-148a. QRT-PCR analysis of enriched MAFB mRNA levels after Ago2 immunoprecipitation. IgG was used as negative control $(n=3)$. (F) Dual-luciferase reporter assays showing direct interaction of miR-148a with its cognate binding sites in the $3^{\prime} U T R$ of MAFB. MAFB $3^{\prime} U T R$ mutant refers to construct with mutated cognate binding sites for miR-148a. The renilla luciferase (Renilla) signal regulated by the target genes 3'UTR was normalized by the firefly luciferase (Firefly). The Renilla/Firefly ratio was set as 1 for the control psiCHECK2 plasmid $(n=3)$. (G) Knockdown of MAFB reduced the difference in the differentiation of moDCs between WT and miR-148a $a^{-/-}$monocytes. Monocytes derived from WT and miR-148a-- mice were transfected with control (NC) or MAFB siRNA and then cultured with GM-CSF and IL-4 for indicated days. The differentiation of moDCs was analyzed $(n=3)$. Statistical analysis was performed with 2-tailed unpaired $t$ test or 1-way ANOVA with Bonferroni's post hoc test. Data are shown as mean \pm SEM $\left({ }^{*} P<0.05,{ }^{* *} P<0.01,{ }^{* *} P<0.001\right.$, n.s., not significant). miR-148a, microRNA-148a. 
A

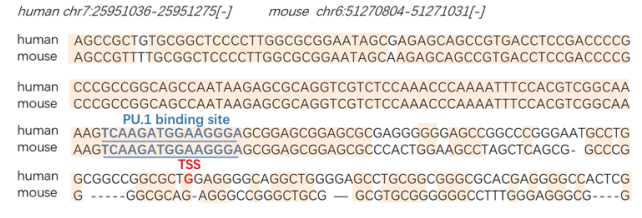

B

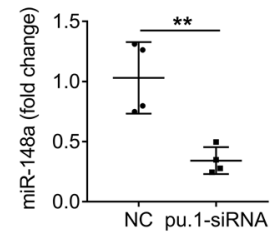

C $\rightarrow$ pu. 1

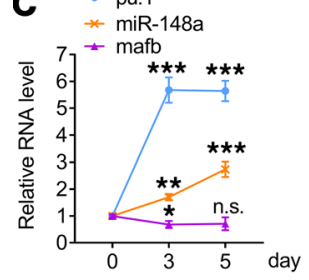

$\mathbf{F}$

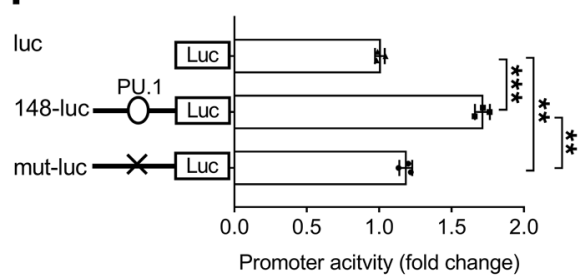

E

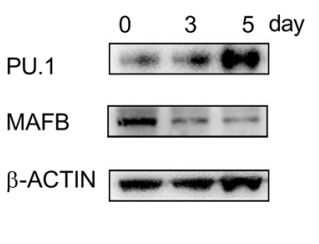

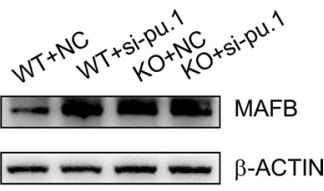

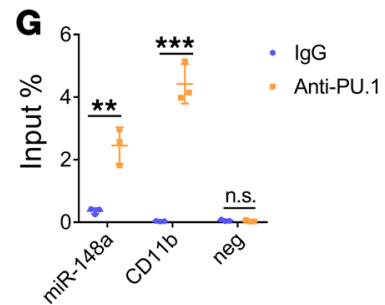

Figure 3. miR-148a is involved in the inhibition of MAFB expression by PU.1. (A) Sequence alignment of the human and mouse miR-148a promoter (-strand) showing localization of predicted PU.1 binding site (blue). The transcriptional start sites are highlighted in red. Genomic locations for the human (GRCh38/hg18) and mouse (GRCm38/mm10) sequence intervals are provided above. (B) TaqMan qRT-PCR analysis of miR-148a expression in control (NC) or PU.1 siRNA transfected human monocytes. U6 snRNA was used as endogenous control $(n=3)$. (C and D) Kinetic analysis of miR-148a, PU.1 and MAFB expression during moDC differentiation. (C) mRNA levels of PU.1 and MAFB were detected by qRT-PCR and normalized to the $\beta$-ACTIN RNA level. The $m R N A / \beta$-ACTIN ratio on day 0 was set as 1 . (D) Immunoblot analysis. The protein $/ \beta$-ACTIN ratio on day 0 was set as 1 . (E) Monocytes isolated from WT and $m i R-148 a^{-/-}$BM cells were transfected with control (NC) or siRNA specific to PU.1 (si-PU.1), and the protein levels of MAFB were detected. The MAFB/ $\beta$-ACTIN ratio in WT+NC was set as 1. (F) Luciferase reporter assay in Hela cells cotransfected with the promoter of miR-148a (148-luc) or miR-148a promoter lacking the PU.1 binding site (mut-luc), and a vector overexpressing PU.1. The firefly luciferase signal was normalized by renilla luciferase. The Firefly/Renilla ratio was set as 1 for the no promoter empty plasmid (luc) ( $n=3$ ). (C) Binding of PU.1 within the miR-148a promoter was analyzed using ChIP and qRT-PCR on human monocytes collected on day 0 or day 3 during moDC differentiation. The ets site in the CD11b promoter was set as positive control, whereas the downstream of miR-148 TSS was set as negative control (neg). Data are the mean percentage of input $\pm \operatorname{SEM}(n=3)$. $P$ values compare the indicated groups using a 2-tailed unpaired $t$ test or 1-way ANOVA with Bonferroni's post hoc test. Data are shown as mean $\pm \operatorname{SEM}\left({ }^{*} P<0.05,{ }^{*} P<0.01,{ }^{* *} P<0.001\right.$, n.s., not significant). miR-148a, microRNA-148a; moDC, monocyte-derived DC; quantitative real-time PCR, qRT-PCR.

$m i R-148 a$ was indispensable for the inhibition of MAFB expression by PU.1.

PU.1 directly activates miR-148a promoter. To investigate the role of PU.1 in directly regulating $m i R-148 a$ expression, we generated luciferase reporter constructs containing a 200-bp region of the human miR-148a promoter. As shown in Figure 3F, overexpression of PU.1 enhanced miR-148a promoter activity compared with that for cells transfected with an empty vector. Additionally, deletion of the PU.1 binding site significantly attenuated the promoter activity.

To determine whether PU.1 binds to this site in monocytes, we performed the ChIP assays. PU.1chromatin complexes were immunoprecipitated from monocytes with a PU.1 monoclonal antibody, and qPCR with site-specific primers was designed to analyze for enrichment of the identified site. Clear PU.1 occupancy was observed for the putative binding site within the promoter region of miR-148a (Figure $3 \mathrm{G}$ ).

In summary, these researches demonstrated that PU.1 inhibited MAFB expression through transcriptional activation of $m i R-148$ a directly.

$m i R-148$ a deficiency alleviates the development of psoriasis. To investigate the functional relevance of $m i R-148 a$ in the development of moDCs, we subjected $m i R-148 a^{-/-}$mice to the IMQ-induced psoriasis-like mouse model. Repetitive application of IMQ onto WT mouse skin led to psoriasiform inflammation with significant thickening, redness, and scaling caused by keratinocyte hyperproliferation and leukocyte infiltration into the skin (Figure 4, A-F). Compared with WT mice, $m i R-148 a^{-/-}$mice 
developed much less skin inflammation; exhibited less thickening, redness, and scaling (Figure 4A); displayed a greater reduction in ear thickness (Figure 4B); and had a lower Psoriasis Area and Severity Index (PASI) score (Figure 4C). H\&E-stained back skin sections of $m i R-148 a^{-/-}$mice displayed a reduction in dermal thickness and acanthosis (Figure 4, D-F) during the course of disease. On day 6 , the peak of psoriasis-like changes, we observed that the splenomegaly of mice in the $m i R-148 a^{-/-}$group was much less obvious than that in the control group (Supplemental Figure 5, A and B). In accordance with this, the reduced mRNA of $I L 23, T N F \alpha, I L 6, I L 1 \beta$, and $I L 17 a$ was observed in the skin lesions of the

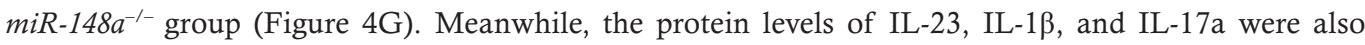
found to be remarkably decreased in the serum of $m i R-148 a^{-1-}$ mice (Figure $4 \mathrm{G}$ ).

To further determine the inflammatory cell infiltration in dermis from the $m i R-148 a^{-/-}$group, we obtained dermal single-cell suspensions and analyzed the percentage of different immune cell subsets. The percentage of $\mathrm{CD}^{4} 5^{+}$immune cells, including moDCs $\left(\mathrm{CD} 45^{+} \mathrm{MHCII}{ }^{\mathrm{hi}} \mathrm{Ly} 6 \mathrm{C}^{-} \mathrm{CD} 64^{-} \mathrm{CD}\right.$ $\left.11 \mathrm{c}^{+} \mathrm{CD} 11 \mathrm{~b}^{+}\right)$and $\mathrm{IL}-17^{+} \gamma \delta \mathrm{T}$ cells $\left(\mathrm{CD} 45^{+} \mathrm{CD}^{+} \gamma \delta \mathrm{TCR}^{+} \mathrm{IL}-17 \mathrm{~A}^{+}\right)$, was greatly reduced in dermis (Supplemental Figure 5C, and Figure 4, H and I); in contrast, macrophages (CD45 ${ }^{+} \mathrm{MHCII}^{+} \mathrm{Ly}_{6 \mathrm{C}}{ }^{-} \mathrm{CD} 64^{+}$) increased (Figure 4, H and I). Similarly, the number of splenocytes, B cells, Ly6C $\mathrm{Ch}^{\text {hi }}$ monocytes, moDCs,

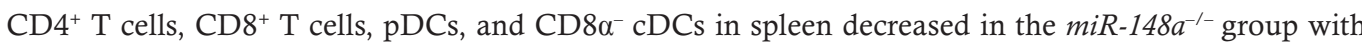
IMQ application (Supplemental Figure 5, B and D). Meanwhile, both CD $8 \alpha^{+}$CDCs and neutrophils in spleen were not affected (Supplemental Figure 5D). To determine whether moDCs reduced in the skin lesions of $m i R-148 a^{-1-}$ mice, we also performed the IHC assay on skin sections using an antibody specific for mouse CD11c. The results showed that moDCs decreased in the dermis of miR-148a ${ }^{-/-}$mice with respect to those of WT mice (Supplemental Figure 5E). Furthermore, we also detected the expression of $M A F B$ and found that $M A F B$ decreased in this model (Supplemental Figure 5F).

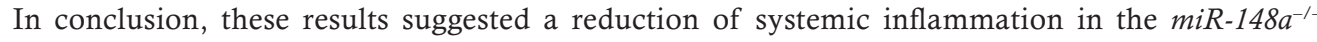
group during the psoriasis.

$m i R-148$ in moDC contributes to inflammation. To confirm whether miR-148a exerted an effect on psoriasis through dependence on immune cells, rather than other skin tissue cells, WT or $m i R-148 a^{-1-} \mathrm{BM}$ cells were transplanted into sublethally irradiated $\mathrm{CD} 45.1^{+}$recipients, to generate $\mathrm{BM}$ chimeric mice. Six weeks after transplantation, these mice were subjected to the IMQ-induced psoriasis-like mouse model (Supplemental Figure 6A). Compared with WT mice, $m i R-148 a^{-1-}$ chimeric mice developed much less skin inflammation; exhibited less thickening, redness, and scaling (Supplemental Figure 6B) and had lower PASI score and displayed a greater reduction in ear thickness (Supplemental Figure 6C). H\&Estained back skin sections of $m i R-148 a^{-/-}$chimeric mice displayed a reduction in acanthosis and dermal thickness (Supplemental Figure 6, D and E) compared with controls. Meanwhile, we observed that splenomegaly was much less obvious in the $m i R-148 a^{-/-}$chimeric group than the control group (Supplemental Figure 6, F-H). FACS analysis of dermis showed that miR-148a $a^{-1}$ chimeric mice had much less infiltration of $\mathrm{CD} 45^{+}$immune cells, including moDCs and IL-17 $7^{+} \gamma \delta \mathrm{T}$ cells (Supplemental Figure 6I). Similarly, the reduced mRNA levels of IL23a, TNF , and $I L 17 a$ were also observed in the skin lesions of the $m i R-148 a^{-/-}$chimeric group (Supplemental Figure $6 \mathrm{~J}$ ).

To further assess that the role of $m i R-148 a$ was moDC intrinsic, we adopted antibody-mediated depletion of

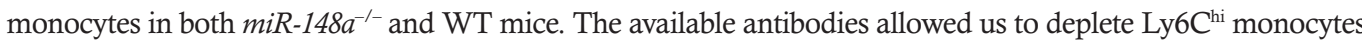
plus neutrophils or neutrophils alone (Figure 5A and Supplemental Figure 7A). Depleting neutrophils alone had little effect on the inflammatory response and psoriasis progression (Figure 5, B-G), which was consistent with a previous report (26). The additional depletion of Ly6 $\mathrm{C}^{\text {hi }}$ monocytes led to a partial loss of infiltrating monocytes and moDCs (Figure 5G and Supplemental Figure 7A), accompanied by a significant decrease in ear swelling, PASI score (Figure 5C), dermal thickness, and acanthosis (Figure 5, D and E) as well as in mRNA levels of $I L 23 a, T N F \alpha, I L 6$, and $I L 17 a$ (Figure 5F). More crucial, there was no difference in the severity of psoriasis-like skin inflammation between IMQ-painted $m i R-148 a^{-/-}$and WT mice after Ly6C ${ }^{\text {hi }}$ monocyte depletion, since they showed a similar reduction in ear and epidermal thickness (Figure 5, B-E) and in moDC and IL- $17^{+} \gamma \delta \mathrm{T}$ cell infiltration (Figure 5G). The difference in the mRNA level of the inflammatory cytokines also greatly diminished (Figure 5F). In accordance with these findings, the difference in the splenomegaly and moDC number

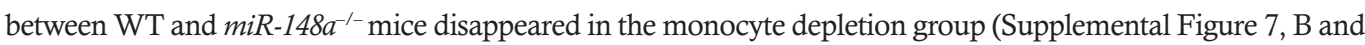
C). Consequently, the fact that the depletion of monocytes eliminated the acceleratory effects of miR-148a on the development of psoriasis suggested that the role of $m i R-148 a$ was more likely moDC intrinsic.

Intradermal administration of antagomir-148a ameliorates the pathological phenotype of IMQ-induced psori- 
A

Control

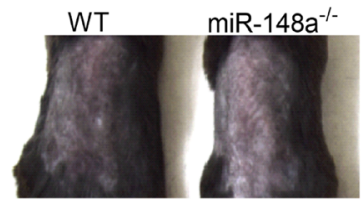

IMQ

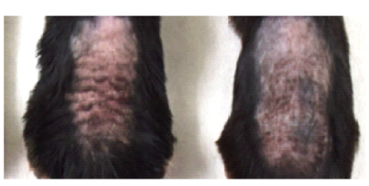

D

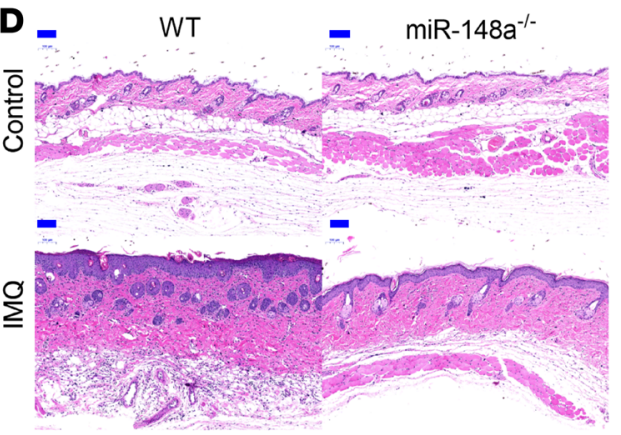

B

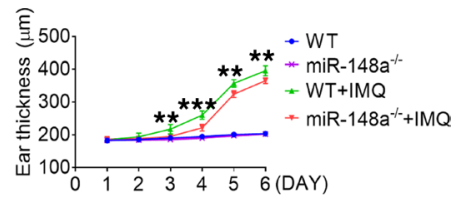

C

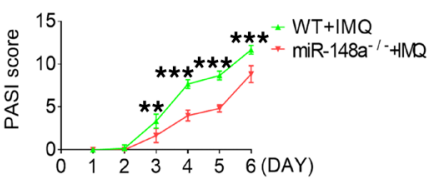

E

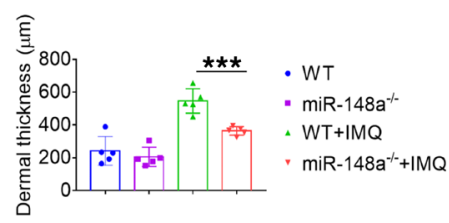

$\mathbf{F}$

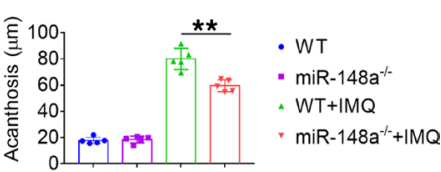

G

IL-23

TNF- $\alpha$

IL-6

IL-1 $\beta$

IL-17a
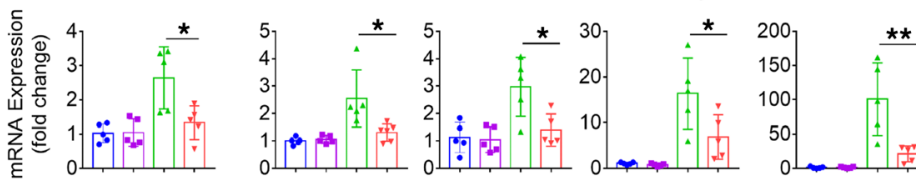

- WT

- $\operatorname{miR}-148 \mathrm{a}^{-/-}$
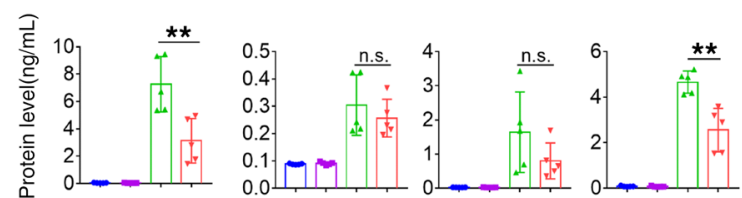

- $W T+I M Q$
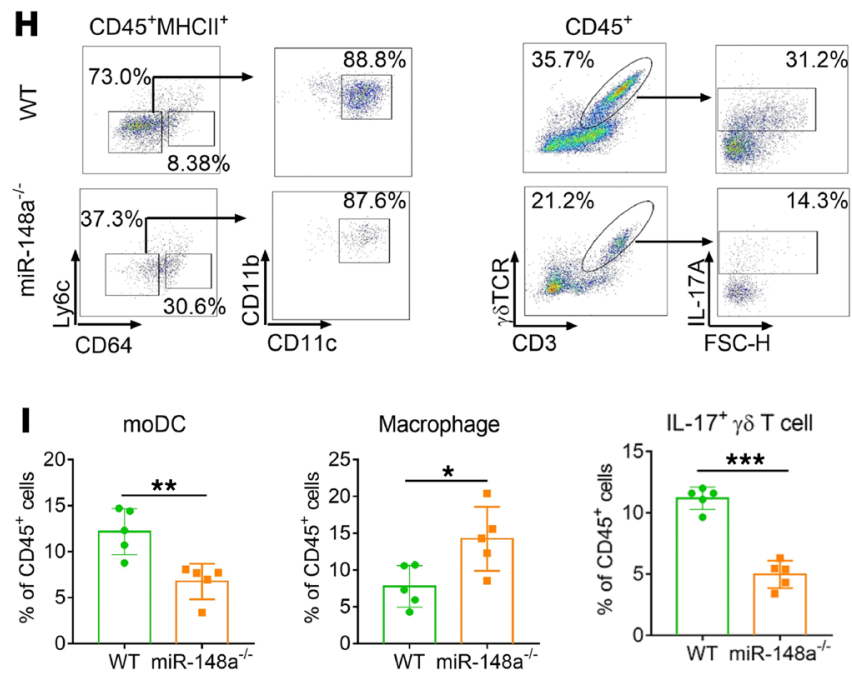

Figure 4. miR-148a deficiency alleviates the development of psoriasis. WT and $m i R-148 a^{-/-}$mice were treated with IMQ for 6 consecutive days. (A) Phenotypic presentation of skin lesions of representative mice on day 6. (B) Ear thickness. (C) PASI score. (D) H\&E staining. (E) Dermal thickness. (F) Acanthosis. $(n=5)$. (C) The relative mRNA expression of indicated cytokines in skin (top) and the protein levels (bottom) of these cytokines in serum on day $4(n=5)$. (H) Flow cytometry analysis of the

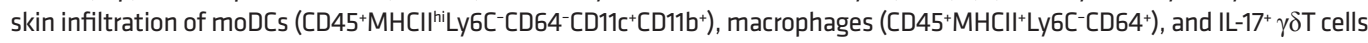
$\left(\mathrm{CD}_{4}{ }^{+} \mathrm{CD}^{+} \gamma \delta \mathrm{TCR}^{+} \mathrm{IL}-17 \mathrm{~A}^{+}\right)(n=5)$. (I) Statistical data of the skin infiltration of moDCs, macrophages, and IL-17+ $\gamma \delta \mathrm{T}$ cells $(n=$ 5). Scale bars: $100 \mu \mathrm{m}$ (D). $P$ values were determined by a 2 -tailed unpaired $t$ test. Data are shown as mean $\pm \operatorname{SEM}\left({ }^{*} P<0.05\right.$, ${ }^{* *} P<0.01,{ }^{* *} P<0.001$, n.s., not significant). miR-148a, microRNA-148a; IMQ, imiquimod; moDC, monocyte-derived DC; PASI, Psoriasis Area and Severity Index. 
A

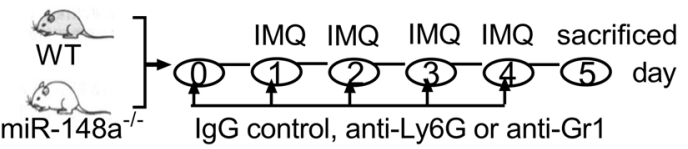

B

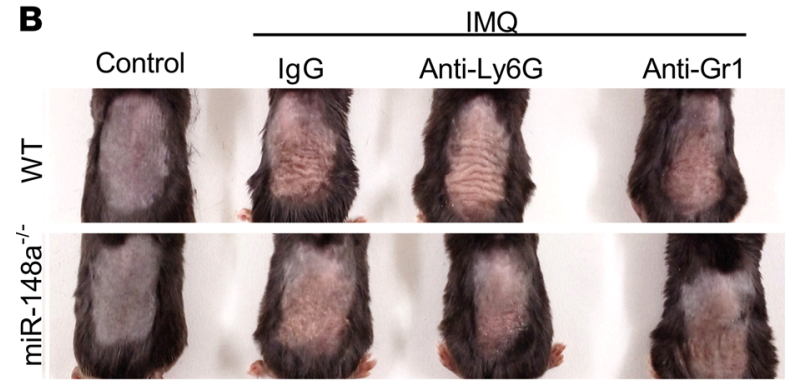

D

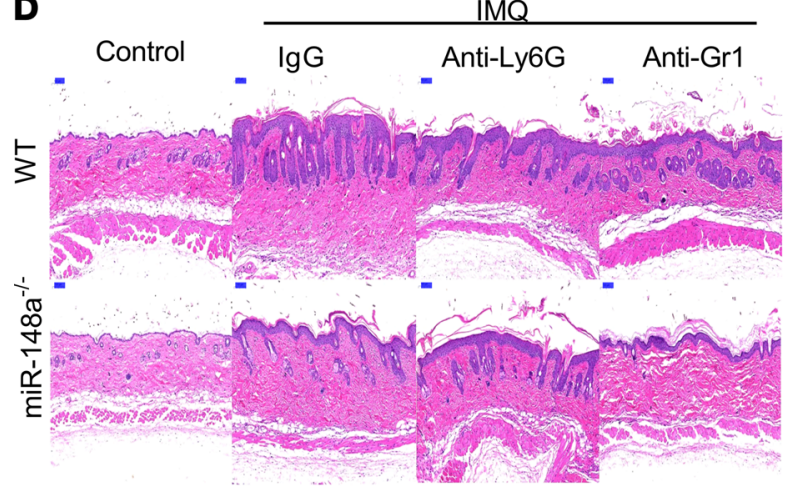

IL-23a

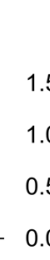

TNF- $\alpha$
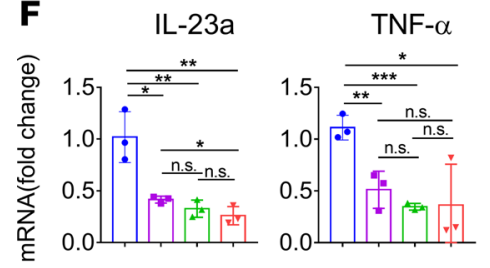
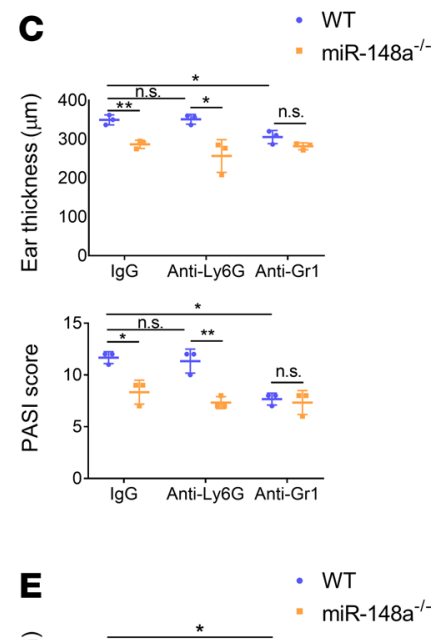
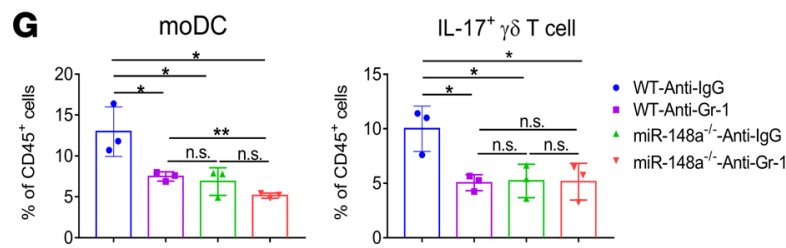

Figure 5. moDC intrinsic miR-148a contributes to inflammation. (A) Schematic diagram for IMQ application and depletion of monocytes plus neutrophils or neutrophils alone in WT or $m i R-148 a^{-1-}$ mice. Phenotypic presentation (B) and H\&E staining (D) of skin lesions of representative mice on day $5(n=3)$. Decrease in ear thickness (C, top), PASI score (C, bottom), dermal thickness (E, top), and acanthosis ( $\mathbf{E}$, bottom) on day $5(n=3)$. (F) The relative mRNA expression of indicated cytokines in skin $(n=3)$. (G) Statistical data of the skin infiltration of moDCs and IL-17+ $\gamma \delta$ T cells $(n=3)$. Scale bars: $100 \mu \mathrm{m}$ (D). $P$ values were determined by a 2-tailed unpaired $t$ test or by 1-way ANOVA with Bonferroni's post hoc test. Data are shown as mean $\pm \mathrm{SEM}\left({ }^{*} P<0.05,{ }^{* *} P<0.01,{ }^{* * *} P<0.001\right.$, n.s., not significant). moDC, monocyte-derived DC; miR-148a, microRNA-148a; PASI, Psoriasis Area and Severity Index.

asis-like mice. The above-mentioned results indicated that elevated miR-148a expression could promote psoriasis pathogenesis. Therefore, we next sought to evaluate the efficacy and potential application of a miR-148a-based therapy for psoriasis. Antagomir-148a or antagomir-NC was intradermally injected 4 times from the beginning to the third day to test the inhibitory effect on disease development in this model (Figure 6A). miR-148a expression in skin lesions was dramatically reduced (Supplemental Figure $8 \mathrm{~A})$. The results displayed a significant improvement in both clinical and pathological characteristics on day 5 after antagomir-148a treatment (Figure 6, B-E), along with alleviated disease severity (Figure 6C) and splenomegaly (Supplemental Figure 8, B and C). In addition, IMQ-treated mice injected with antago- 
mir-148a manifested lower levels of $I L 23 a, T N F \alpha, I L 6, I L 1 \beta$, and $I L 17 a$ mRNA in skin lesions (Figure 6F). We also assessed the effect of antagomir-148a in vivo on moDCs and IL-17+ $\gamma \delta \mathrm{T}$ cells in psoriasis-like mouse models. Silencing of $m i R-148 a$ significantly reduced the percentage of moDCs and IL- $17^{+} \gamma \delta \mathrm{T}$ cells in skin lesions (Figure 6, G and $\mathrm{H}$ ). In accordance with the decrease of $M A F B$ in the IMQ-induced model (Supplemental Figure 5F), mice with antagomir-148a administration showed higher levels of $M A F B$ than mice with antagomir-NC (Supplemental Figure 8D).

Because it has been demonstrated that $m i R-148$ a could regulate moDC differentiation by directly targeting $M A F B$ in vitro, it is crucial to confirm the mechanism in vivo. Accordingly, $m i R-148 a^{-/-}$and WT mice were administered specific siRNA for $M A F B$ or NC and painted with IMQ for 6 days. Then, the knockdown efficiency was verified (Supplemental Figure 9A). Compared with mice with NC siRNA, those with $M A F B$ siRNA showed a significant aggravation in both clinical and pathological characteristics (Supplemental Figure 9, B-E), along with exacerbated disease severity (Supplemental Figure 9C) and splenomegaly (Supplemental Figure 9F). Furthermore, MAFB knockdown weakened the effect of $m i R-148 a$ deficiency, because the difference between WT and $m i R-148 a^{-1-}$ mice narrowed in the group treated with $M A F B$ siRNA, compared with groups treated with NC (Supplemental Figure 9, B-F). Thus, MAFB partially mediated the function of $m i R-148 a$ in vivo.

Finally, to examine the relevance of the expression level of miR-148a to psoriasis disease, we detected the expression level of $m i R-148 a$ in peripheral monocytes from patients with psoriasis. Compared with those from healthy controls, there was an increase in mRNA levels of $I L 23 a, I L 1 \beta$, and $I L 17 a$ in PBMCs from psoriatic patients (Figure 7A). Monocytes from patients with psoriasis showed an increased expression level of $m i R-148 a$ (Figure 7B). The expression level of $m i R-148 a$ positively correlated with the mRNA levels of $I L 23 a$ and $I L 17 a$ (Figure 7C), supporting that miR-148a was crucial in psoriasis. To confirm the PU.1-miR-148a-MAFB circuit in psoriasis in vivo, we detected the expression of PU.1 and MAFB in peripheral monocytes from patients with psoriasis. The results demonstrated that monocytes from patients with psoriasis showed a decreased expression level of $M A F B$ but an increased expression level of PU.1 (Figure 7, D and E). Moreover, the expression level of miR-148a positively correlated with the mRNA level of PU.1 (Figure 7E) and negatively correlated with $M A F B$ level (Figure 7D).

Consequently, these results indicated that the inhibition of $m i R-148 a$ expression in vivo could prevent the immunopathological changes in psoriasis and effectively ameliorate disease severity, suggesting a novel treatment strategy for psoriasis and other autoimmune diseases.

\section{Discussion}

The transcriptional regulation of moDC differentiation has been extensively studied $(2,13)$. The balance of MAFB and PU.1 was considered to specify alternative macrophage or DC fate (18). The differentiation of monocytes to DCs results in a rapid increase of PU.1, achieving high levels that precede the phenotypic changes, while MAFB expression is decreased during the differentiation. Inhibited MAFB expression in monocytes appears to be required for DC specification, since constitutive MAFB expression inhibits DC differentiation (18). In addition, $M A F B$ deficiency is found to specifically enhance sensitivity to M-CSF and cause activation of the myeloid master regulator PU.1 in hematopoietic stem cells in vivo (19). One possible mechanism for the PU.1 regulation of MAFB function is that PU.1 can directly bind to MAFB and inhibit MAFB transactivation capacity. In this research, we found that miR-148a mediated the inhibition of MAFB expression through PU.1 regulation, suggesting a new mechanism for regulating the balance of MAFB and PU.1 and, thereby, the differentiation of moDCs. Interestingly, a recent study reported that PU.1 could directly bind to the superenhancer of $m i R-148 \mathrm{a}$ and facilitate its maturation in mouse primary B cells (36). Whether this regulation exists in primary monocytes and moDCs remains to be made clear.

miRNA is generally regarded as a multifunctional molecule and can exert diverse roles in different immune cells by targeting different molecules intracellularly (28). Increased expression of miR-148a, which occurs frequently in patients with lupus and lupus-prone mice, impaired B cell tolerance through promoting the survival of immature B cells upon B cell receptor engagement via suppressing the expression of GADD45a, PTEN, and BCL2111 - and facilitated the development of lethal autoimmune disease (33). miR-148a could also considerably reduce the DNMT1 protein level, which was one of the major epigenetic components that linked to DNA hypomethylation in T cells in SLE (31). There was no significant difference in the number of $\mathrm{B}$ cell and $\mathrm{T}$ cell subsets between $m i R-148 a^{-/-}$mice and WT mice in the steady state. Hence, miR-148a might affect the survival and function of both autoreactive B and $\mathrm{T}$ 
A

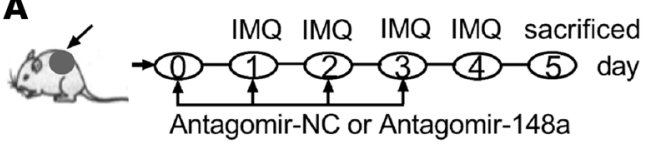

B

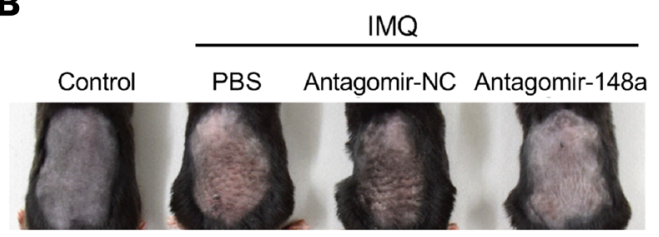

D
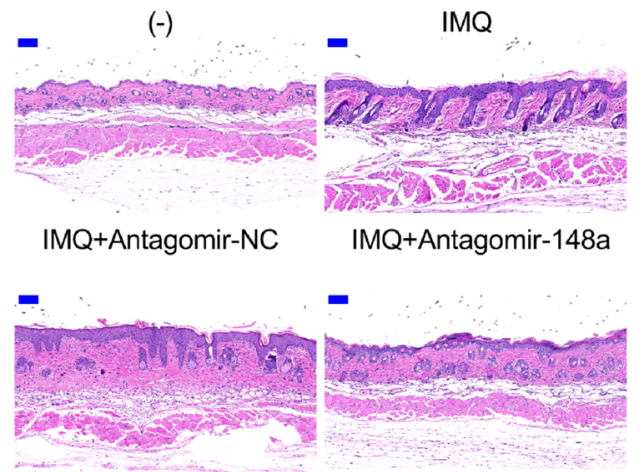

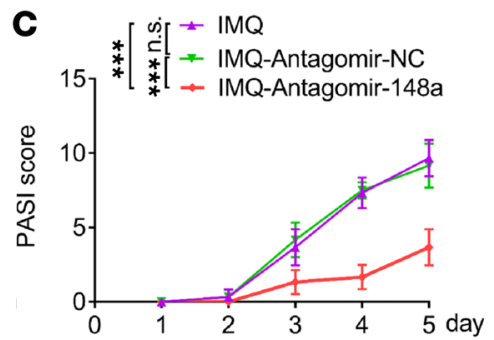

E

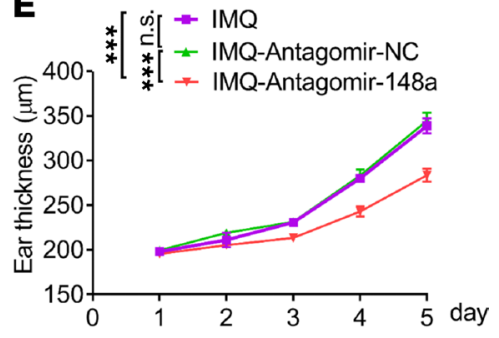

Figure 6. Intradermal administration of antagomir-148a ameliorates the pathological phenotype of IMQ-induced psoriasis-like mice. (A) Schematic diagram for IMQ application and intradermal administration of antagomir-NC or antagomir-148a. Phenotypic presentation (B) and H\&E staining (D) of skin lesions of representative mice on day $5(n=6)$. Decrease in PASI score (C) and ear thickness (E) during the course of disease $(n=6)$. (F) The relative mRNA expression of indicated cytokines in skin $(n=6)$. Flow cytometry analysis (G) and statistical data (H) of the skin infiltration of moDCs and IL-17+ $\gamma \delta$ T cells $(n=6)$. Scale bars: $100 \mu \mathrm{m}$ (D). $P$ values were determined by a 2-tailed unpaired $t$ test or 1-way ANOVA with Bonferroni's post hoc test. Data are shown as mean \pm SEM $(* P<0.05$, ${ }^{* *} P<0.01,{ }^{* *} P<0.001$, n.s., not significant). IMQ, imiquimod; PASI, Psoriasis Area and Severity Index.
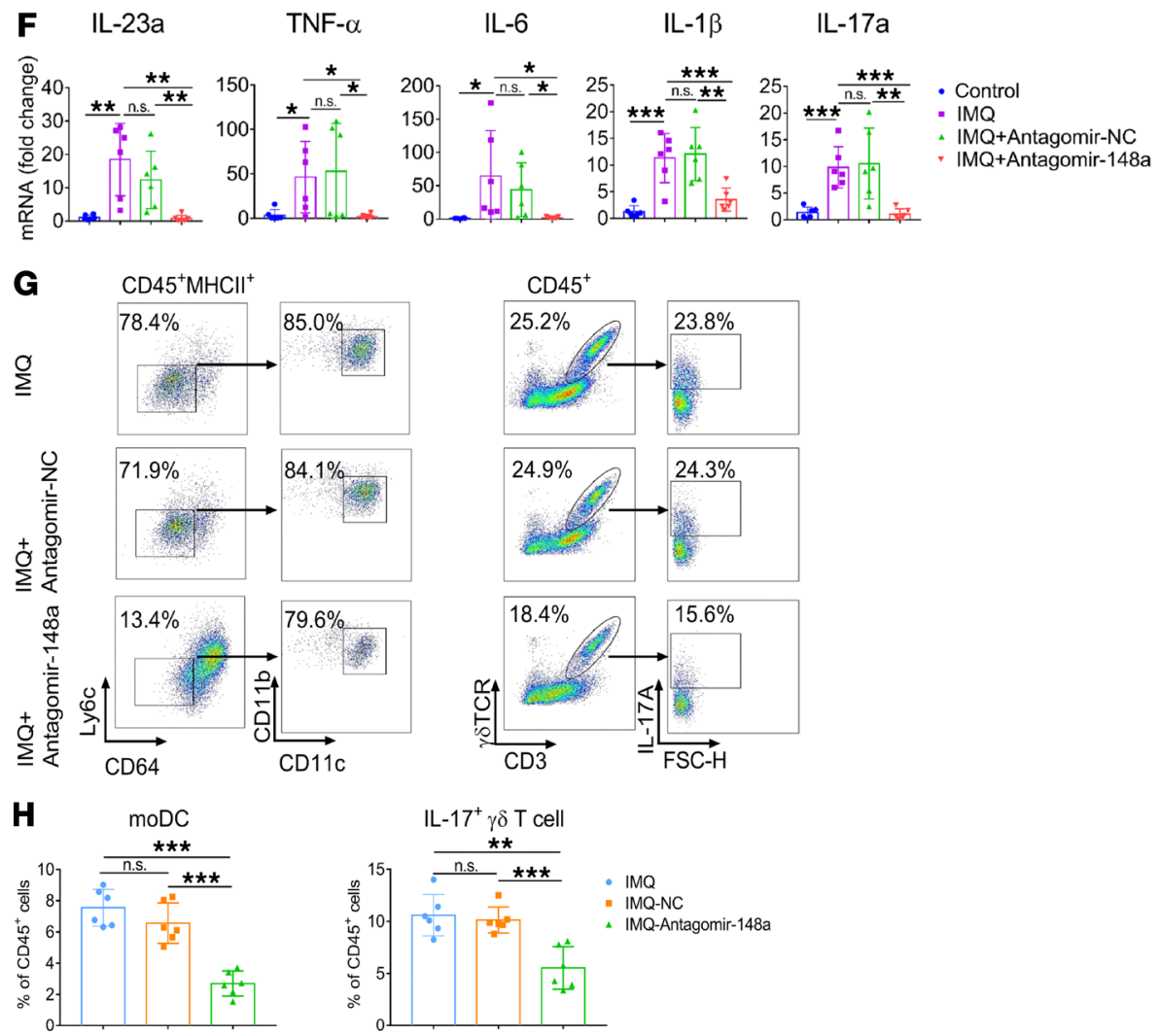

cells in the inflammatory state. To confirm the cell-intrinsic role of $m i R-148 a$ in moDCs, we designed two experiments. First, we constructed BM chimeric mice and found that $m i R-148 a^{-1-}$ chimeric mice demonstrated less psoriatic skin inflammation, indicating that miR-148a takes effect only on immune cells, rather than other skin tissue cells during the process. Second, monocytes were depleted in vivo through the use of antibodies before and during IMQ painting. Monocyte depletion greatly alleviated the severity 

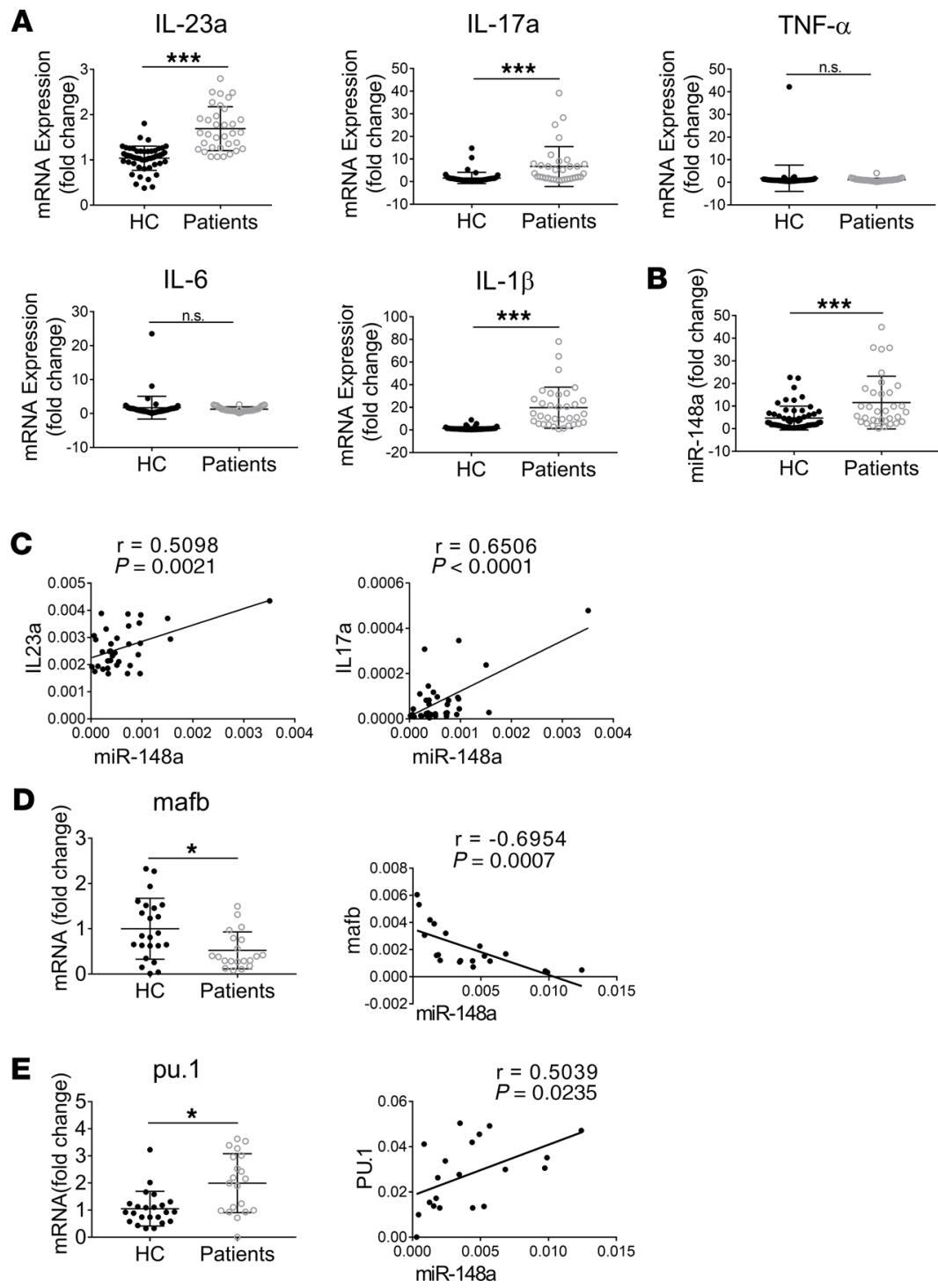

Figure 7. The expression of miR-148a is elevated in psoriatic monocytes. (A) Proinflammatory cytokine gene expression in the PBMCs derived from patients with psoriasis $(n=34)$ and healthy controls $(n=51)$. (B) Expression of miR-148a in peripheral monocytes isolated from patients with psoriasis $(n=34)$ and healthy controls $(n=51)$. (C) The correlation analysis between the miR-148a level in psoriatic monocytes and the mRNA level of IL23a and IL17a in psoriatic PBMCs $(n=34)$. ( $\mathbf{D}$ and $\mathbf{E})$ Expression of MAFB (D) and PU.1 (E) in peripheral monocytes isolated from patients with psoriasis ( $n$ $=20$ ) and healthy controls $(n=23)$, and the correlation analysis between the miR-148a and MAFB (D) or PU.1 (E) level in psoriatic patients $(n=20)$. $P$ values compare the indicated groups using nonparametric Mann-Whitney $U$ test or Spearman's test. Data are shown as mean \pm SEM $\left({ }^{*} P<0.05,{ }^{* *} P<0.001\right.$, n.s., not significant). miR-148a, microRNA-148a.

of the psoriasiform skin inflammation. In addition, there was no significant difference in the symptoms between $m i R-148 a^{-/}$mice and WT mice, indicating that miR-148a in moDCs is more likely to be critical for the IMQ-induced psoriasis-like inflammation.

It has also been reported that miR-148a regulates the innate immune response. It has been demonstrated that miR-148a negatively regulates the TLR4-triggered functional maturation of moDCs by targeting CaMKIIa (34), and inhibits macrophage function by directly targeting several upstream regulators of NF- $\mathrm{kB}$ and STAT3

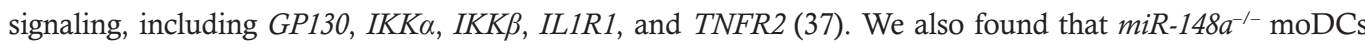
could produce increased levels of TNF- $\alpha$ and IL-6 in response to LPS stimulation (data not shown). However, a less than $10 \%$ increase was rather limited compared to the decreased potential of moDC differentiation both in vitro (about 70\% reduction) and in vivo (a more than 1-fold reduction), as shown in Figure 1C, Figure 4I, and Figure $6 \mathrm{H}$. Therefore, miR-148a in monocytes exerted deleterious effects during psoriasis.

In addition to miR-148a, several other miRNAs have been reported to regulate the differentiation and 


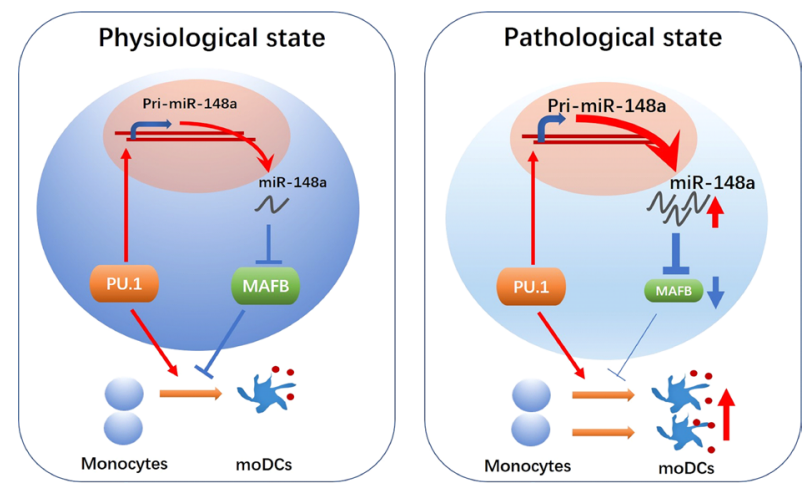

Figure 8. Regulation model. miR-148a, which could be transcriptionally activated by PU.1, promotes moDC differentiation by directly targeting MAFB, thereby causing the immune imbalance and inflammatory response. miR-148a, microRNA-148a; moDC, monocyte-derived DC.

function of moDCs, such as $m i R-21, m i R-34 a$, and $m i R-155$ (38-41). Besides miR-148a, both $m i R-21$ and $m i R-34 a$ also affect moDC generation, and miR-155 promotes the apoptosis and activation of moDCs. As molecule fine-tuning gene expression, these miRNA targets generate a complex regulatory network that comprise different levels, including $m i R-21 / W N T 1$ and $m i R-34 a / J A G 1$ at the ligand/receptor level, $m i R-155 /$ SOCS1 at the signal transduction level, and miR-148a/MAFB at the transcriptional factor level. Interestingly, $m i R-21$ was found to be abnormally elevated in psoriasis, and the inhibition has been shown to have a beneficial effect on the treatment of psoriasis (42). In addition, miR-155 is also upregulated in psoriatic biopsy samples and responsible for maintaining the inflammation in psoriasis (43).

In conclusion, the present study provides evidence that miR-148a, which could be transcriptionally activated by PU.1, promotes moDC differentiation through directly targeting $M A F B$, thereby causing the immune imbalance and inflammatory response in skin lesions of psoriasis (Figure 8). Since inflammatory DCs are pathogenic in various autoimmune diseases (SLE, RA, etc.), this research suggests that miR-148a could serve as a potential target for the treatment of not only psoriasis but also other autoimmune diseases.

\section{Methods}

Human subjects. A total of 34 psoriatic patients, who were diagnosed with psoriasis vulgaris, were recruited from outpatient clinics at Huashan Hospital of Fudan University. Psoriasis disease activity was assessed using PASI score. The patient information is shown in Supplemental Table 1. In addition, a total of 51 sexand age-matched healthy controls were recruited from Renji Hospital of SJTUSM.

Mice. The C57BL/6J mice were acquired from Slack Company. miR-148a-knockout mice B6/JNjuMir148a $\mathrm{a}^{\mathrm{em} 2 \mathrm{Cd} 26 \mathrm{in} 1} / \mathrm{Nju}$ on a [C57BL/6] background were provided by Model Animal Research Center, Nanjing University. In addition, male mice at $6-8$ weeks of age were used to carry out all the following experiments. All mice were bred and housed under the conditions of specific pathogen-free circumstance.

$I M Q-i n d u c e d$ psoriasis-like mouse model and antagomir-148a and MAFB siRNA treatment. Male miR-148a-1mice on a C57BL/6J background (6-8 weeks of age) were daily painted with IMQ cream (5\%) (Aldara; $3 \mathrm{M}$ Pharmaceuticals) on the shaved back for the indicated days and then sacrificed for the subsequent experiments. The control mice were applied with the same dose of vehicle cream. Male $m i R-148 a^{-1-}$ mice and their littermates (6-8 weeks of age) were injected intradermally with antagomir-148a (10 nM in 150 $\mu \mathrm{L}$ PBS) or antagomir-NC (10 nM in $150 \mu \mathrm{L}$ PBS) on days $0,1,2$, and 3 during the application of IMQ (44). Antagomir-148a (GenePharma, B05001) and the corresponding negative control antagomir-NC (GenePharma, B04007) were purchased from Shanghai GenePharma Co. Ltd. As for MAFB siRNA

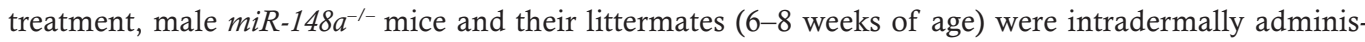
tered siRNA specific for MAFB (10 $\mathrm{nmol}$ in $100 \mu \mathrm{L}$ PBS) or NC (10 nmol in $100 \mu \mathrm{L}$ PBS) on days 0,1 , 2 , and 3 during the application of IMQ. In addition, $M A F B$ siRNA (Ambion, 4457308) and NC siRNA (Ambion, 4457289) were purchased from Applied Biosystems.

Scoring severity of skin inflammation in psoriasis-like mouse model. An objective scoring system was developed based on the clinical PASI for assessing the patients with psoriasis. Accordingly, erythema, thickening, and scaling were each scored according to the scope (0-4: 0 , none; 1 , slight; 2 , moderate; 3 , marked; 
4, very marked). The overall score was obtained by calculating a sum of the 3 index scores (score 0-12).

Cell isolation, flow cytometry analysis, and cell culture. Skin cells were prepared on the basis of previous research with minor modifications (45). In brief, the epidermis and dermis were separated by adopting the dispase (25 U/ml in Dulbecco's DPBS) and then the dermal cell suspension was acquired using collagenase and hyaluronidase. Splenocytes were prepared by digesting the tissue fragments with collagenase type IV and DNAse I. Antibodies used for cell staining were presented in Supplemental Table 2. Cells were analyzed using Fortessa (BD Biosciences) and FlowJo software.

PBMCs were separated from the peripheral blood of healthy controls and patients with psoriasis by density gradient centrifugation (GE Healthcare). Human and mouse monocytes were isolated with the Human Monocyte Isolation Kit II and Mouse Monocyte Isolation Kit (BM) (Miltenyi Biotec), respectively. Then, the cells were cultured in RPMI 1640 medium (Gibco) with 10\% FBS (Gibco) at $37^{\circ} \mathrm{C}$ and $5 \% \mathrm{CO}_{2}$, or collected for subsequent experiments. HEK 293T cells and HeLa cells were maintained in DMEM (Gibco) supplemented with 10\% FBS (Gibco). To obtain moDCs, BM cells or

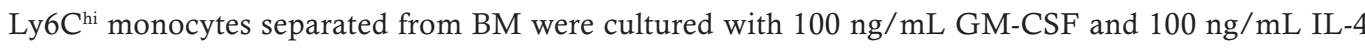
(Peprotech) for indicated days. The Annexin V Apoptosis Detection Kit (BioLegend) was used for apoptosis assays according to the manufacturer's protocol.

siRNAs and agomir transfection. BM cells, mouse monocytes isolated from BM, or human monocytes isolated from peripheral blood were transfected with Lipofectamine RNAiMAX (Invitrogen) according to the protocol. Hela cells and HEK 239T cells were transfected with Lipofectamine 3000 (Invitrogen). Briefly, small interfering RNA (siRNA; $200 \mathrm{nM}$ ), agomir (200 nM), plasmids, and transfection reagents were diluted with Opti-MEM (Gibco) and then gently mixed. After incubation for 15 minutes at room temperature, the transfection mixture was added to the cell culture. Cells were harvested for subsequent experiments. The agomir-148a (GenePharma, B06002) and the corresponding negative control (GenePharma, B04008) were purchased from Shanghai GenePharma Co. Ltd. Human PU.1 siRNA (Santa Cruz, sc-36330), mouse PU.1 siRNA (Santa Cruz, sc-36331), and $M A F B$ siRNA (Santa Cruz, sc-35840) were purchased from Santa Cruz Biotechnology.

Histology. As for H\&E staining, the histological analysis was conducted as Lai et al. reported (46). First, mouse skin tissues were fixed in formalin and embedded in paraffin and then stained with $\mathrm{H} \& \mathrm{E}$. Finally, to assess dermal thickness, we measured the thickness from the stratum papillare to the stratum reticulare and the epidermal hyperplasia from the basal layer to the stratum corneum, using Case Viewer software (3DHISTECH Ltd). The measurement of skin thickness was conducted on 6 randomly selected areas per $\times 10$ field from $3-5$ fields per section. The obtained skin thickness was first averaged per mouse and then used to calculate the average per treatment group for the following statistical analysis. As for immunochemistry, the mouse skin paraffin sections were stained with hematoxylin and mouse monoclonal anti-CD11c antibody (GB11059, Servicebio).

Construction of BM chimeras. B6.CD45.1 recipients were sublethally irradiated by X-ray (8 Gy) and then intravenously transferred with $5 \times 10^{6} \mathrm{BM}$ cells derived from $m i R-148 a^{-/-}$mice or C57BL/6J mice. Chimeras were adopted for experiments 6 weeks after the initial reconstitution.

Depletion of neutrophils and monocytes. The depletion in vivo was conducted based on the report by Singh et al. (26). In brief, as for the depletion of neutrophils alone, the mice were intraperitoneally administered $500 \mathrm{mg}$ antibody anti-Ly6G (clone 1A8, BioXCell) on days $0,1,2,3$, and 4. Meanwhile, to deplete monocytes and neutrophils, the mice were injected intraperitoneally with $500 \mathrm{mg}$ of antibody anti-Gr1 (clone RB6-8C5, BioXCell) at the same time points as above.

$m R N A-S e q$ and miRNA-Seq. All the RNA was first extracted by using the miRNeasy Micro Kit (QIAGEN) and determined with Bioanalyzer 4200 (Agilent). Then, the preparation for the next-generation mRNA libraries was performed with VAHTS mRNA-Seq v2 Library Prep Kit for Illumina (Vazyme), whereas the preparation for the miRNA libraries was performed with NEBNext Multiplex Small RNA Library Prep Set for Illumina (NEB) and then purified by QIAquick PCR Purification Kit (QIAGEN). Afterward, the acquisitions from above were subsequently put into the gel prep for size selection. In addition, the quality of the library was determined using Bioanalyzer 4200 (Agilent). Then, the mRNA-Seq libraries were run in HiSeq X10 system (Illumina) on a paired-end 150-bp run, and the miRNA-Seq libraries were sequenced on an Illumina HiSeq 2500 next-generation sequencer using single-read 50-bp sequencing. Among the compared groups, those with more than 1.2-fold differences of the geometrical mean expression and a statistically significant $P$ value of less than 0.05 based on analysis of DESeq 2 were selected as the differentially expressed genes. To determine statistically enriched GO categories, we conducted the 
following GO analysis of those selected genes using the clusterProfiler R package with $P$ values of less than 0.05. Based on Kyoto Encyclopedia of Genes and Genomes Database (http://www.genome.jp/kegg/), pathway analysis was used to determine the significant pathway of those differential genes. mRNA-Seq and miRNA-Seq data are deposited in the Gene Expression Omnibus repository (GSE142517).

$q R T-P C R$. All the RNA was extracted using Trizol reagent (Invitrogen). Reverse transcription of miRNA to cDNA was implemented with miRNA-specific primers (Applied Biosystems) preceding the qPCR (TaqMan; Applied Biosystems). Noncoding small RNA control U6 (Applied Biosystems) was adopted and served as an endogenous reference gene, with changes in expression calculated by the change in threshold $(\triangle \triangle C T)$ method. For mRNA, cDNA was prepared by reverse transcription (PrimeScript RT Reagent Kit; Takara). The same amounts of cDNA were adopted for the following qPCR conducted with the SYBR PrimeScript Reverse-Transcription PCR kit (Takara). Amplification was implemented through using an ABI PRISM 7900 Real-Time PCR System (Applied Biosystems). The amplification efficiency of these genes was the same as that for $\beta$-ACTIN or RPL13a, as indicated by the standard curves for amplification, allowing us to adopt the subsequent formula, fold difference $=2^{-(\Delta \mathrm{CtA}-\Delta \mathrm{CtB})}$, where $\mathrm{Ct}$ is the cycle threshold. Primer sequences are presented in Supplemental Table 3.

miRNA target prediction. Targetscan version 7.1 (http://www.targetscan.org/mmu_71/) was adopted to predict the miR-148a targets. The detailed descriptions of the complete computational protocol are available at the website and in reference (47).

Luciferase assay. For 3'UTR reporter assays, the 3'UTR of the MAFB mRNA containing WT or mutant miR-148a binding sites were subcloned into the psiCHECK2 vector (Promega). Then, $1 \times 10^{5}$ HEK 293T cells per well were transfected with a mixture of $100 \mathrm{ng}$ 3'UTR luciferase reporter vector and agomirmiR-148a or agomir control using Lipofectamine 2000 (Invitrogen) according to the manufacturer's instructions. Twenty-four hours after transfection, cells were lysed, and luciferase activity was measured on a luminometer (Promega) using the Dual-Luciferase Reporter Assay System (Promega). The ratio of renilla luciferase activity to firefly luciferase activity was calculated for each well.

For promoter reporter assay, $200 \mathrm{bp}$ upstream of the TSS of miR-148a with or without a mutant PU.1 binding sequence were subcloned into the pGL3-basic vector (Promega). Then $1 \times 10^{4} \mathrm{HeLa}$ cells per well were transfected with a mixture of $100 \mathrm{ng}$ pGL3 luciferase vector and $8 \mathrm{ng}$ pRL-TK Renilla vector with or without 100 ng PU.1 expression plasmid or empty control plasmid. Twenty-four hours after transfection, cells were lysed, and luciferase activity was measured on a luminometer (Promega) using the Dual-Luciferase Reporter Assay System. The ratio of firefly luciferase to renilla luciferase was calculated for each well.

ChIP assays. ChIP assays were conducted with a SimpleChIP Plus Enzymatic Chromatin IP (Magnetic Beads) Kit (Cell Signaling Technology). According to the protocol, monocytes were fixed in $1 \%$ formaldehyde and then lysed for 10 minutes on ice. Chromatin was sheared by enzyme digestion and sonication. Then, the cell lysate was immunoprecipitated overnight at $4^{\circ} \mathrm{C}$ with a monoclonal antibody anti-PU.1 (Cell Signaling Technology, 2258). Subsequently, protein G magnetic beads were added, and the obtained mixture was rotated for 2 hours at $4^{\circ} \mathrm{C}$. After washing and elution, cross-links were reversed by heating at $65^{\circ} \mathrm{C}$ for 2 hours. The eluted DNA was purified, and the samples were analyzed by qPCR with input DNA (total chromatin) as an endogenous control. The promoter in $C D 11 b$ and downstream of $m i R-148 a$ TSS region were set as positive or negative control, respectively. ChIP-qPCR primer sequences are listed in Supplemental Table 3.

Ago2 immunoprecipitation. Ago2 immunoprecipitation was carried out according to the protocol provided in the Magna RIP RNA-Binding Protein Immunoprecipitation Kit (Millipore, 17-700). For the RNA-binding protein immunoprecipitation (RIP) lysate preparation, $2 \times 10^{7}$ human primary monocytes were collected by centrifugation at $211 \mathrm{~g}$ for 5 minutes and then lysed with RIP lysis buffer. For the magnetic bead and antibody preparation, purified antibodies $(5 \mu \mathrm{g})$ were incubated with beads for 30 minutes at room temperature using anti-Ago2 antibody (Millipore, 03-110) as the antibody of interest and normal mouse IgG as negative controls, respectively. Supernatant from the RIP lysate was obtained by centrifugation at $12,210 \mathrm{~g}$ for 10 minutes, $100 \mu \mathrm{L}$ of which was incubated overnight at $4^{\circ} \mathrm{C}$ with the bead-antibody complex, and $10 \mu \mathrm{L}$ served as $10 \%$ input for generating a standard curve or for qRT-PCR comparison. Immunoprecipitated RNAs were purified and analyzed by qRT-PCR. RNA immunoprecipitation qPCR primer sequences are listed in Supplemental Table 3.

Immunoblots. Cell lysates were prepared by lysing $2 \times 10^{6}$ monocytes or moDCs in Radioimmune Precipitation Assay Lysis and Extraction Buffer (Thermo Fisher Scientific) and then separated with 10\% SDS- 
PAGE. The separated proteins were transferred to an Immobilon-P PVDF membrane (Millipore), which was subsequently blocked with SuperBlock T20 PBS blocking buffer (Thermo Fisher Scientific). Final1y, the membrane was incubated with rabbit antibodies against MAFB, 1:1000 (Abcam, ab66506), PU.1, 1:1000 (Cell Signaling Technology, 2258), and $\beta$-ACTIN, 1:2000 (Cell Signaling Technology, 4967).

Cytokine detection. Mouse serum was collected at specific time points, and the protein levels of IL-23, IL-17, IL-1 $\beta$, IL-6, and TNF- $\alpha$ were determined with BD Cytometric Bead Array Mouse/Rat Soluble Protein Master Buffer Kit and Flex Sets, according to the instruction manual provided by the manufacture. Briefly, the phycoerythrin-conjugated detection antibodies are mixed with the specific capture beads and then incubated with recombinant protein standards or samples to generate sandwich complexes. Subsequently, the sandwich complexes of each sample ran on a flow cytometer. Standard curves for all analyses were generated through adopting recombinant protein standards to run one standard mixture. Finally, the sample results were analyzed by FCAP Array software (BD Biosciences).

Statistics. Considering the clinical data, nonparametric Mann-Whitney $U$ test was used to draw comparisons between patients and controls, and Spearman's test was adopted to carry out the correlation analysis. For all other experiments, data were analyzed through using Graph Prism 7.0 software (GraphPad), and $P$ values were determined by 2 -tailed unpaired $t$ test or 1-way ANOVA. $P$ values of less than 0.05 were considered significant.

Study approval. Human sample acquisitions were approved by the Review Board of Renji Hospital of SJTUSM and Huashan Hospital of Fudan University. All experiments were conducted in accordance with the declaration of Helsinki Principles. Informed consent was obtained for all procedures. All the animal experiments were performed with the use of protocols approved by the Institutional Animal Care and Use Committee at Renji Hospital of SJTUSM.

\section{Author contributions}

NS, HZ, and YM designed the project. YM and JL performed the experiments, and YM, JL, and HZ analyzed the data. LW, Z. Ye, and Z. Yin analyzed the bioinformatics data. GL established the mouse model. YY, LL, and JD collected the human samples. QS and ZL participated in the transfection experiments. NS, HZ, YM and LW prepared the manuscript.

\section{Acknowledgments}

This study was supported by grants from National Natural Science Foundation of China (31630021, 31600706, 81974252, 31930037, and 81421001), Shenzhen Science and Technology Project (JCYJ20180504170414637), and Sanming Project of Medicine in Shenzhen (SZSM201602087), Shanghai Municipal Key Medical Center Construction Project (2017ZZ01024-002).

Address correspondence to: Haibo Zhou or Nan Shen, Shanghai Institute of Rheumatology, Renji Hospital, School of Medicine, Shanghai Jiao Tong University, 145 Shan Dong Middle Road, Shanghai 200001, China. Phone: 86.021.68385620; Email: hbzhou1984@gmail.com (H. Zhou); nanshensibs@gmail.com (N. Shen).

1. Shortman K, Naik SH. Steady-state and inflammatory dendritic-cell development. Nat Rev Immunol. 2007;7(1):19-30.

2. Merad M, Sathe P, Helft J, Miller J, Mortha A. The dendritic cell lineage: ontogeny and function of dendritic cells and their subsets in the steady state and the inflamed setting. Annu Rev Immunol. 2013;31:563-604.

3. Durai V, Murphy KM. Functions of murine dendritic cells. Immunity. 2016;45(4):719-736.

4. Worbs T, Hammerschmidt SI, Förster R. Dendritic cell migration in health and disease. Nat Rev Immunol. 2017;17(1):30-48.

5. Malissen B, Tamoutounour S, Henri S. The origins and functions of dendritic cells and macrophages in the skin. Nat Rev Immunol. 2014;14(6):417-428.

6. Scott CL, Henri S, Guilliams M. Mononuclear phagocytes of the intestine, the skin, and the lung. Immunol Rev. 2014;262(1):9-24.

7. Kashem SW, Haniffa M, Kaplan DH. Antigen-presenting cells in the skin. Annu Rev Immunol. 2017;35:469-499.

8. Nestle FO, et al. Plasmacytoid predendritic cells initiate psoriasis through interferon-alpha production. J Exp Med. 2005;202(1):135-143.

9. Johnson-Huang LM, McNutt NS, Krueger JG, Lowes MA. Cytokine-producing dendritic cells in the pathogenesis of inflammatory skin diseases. J Clin Immunol. 2009;29(3):247-256.

10. Helft J, et al. GM-CSF mouse bone marrow cultures comprise a heterogeneous population of CD11c(+)MHCII(+) macrophages and dendritic cells. Immunity. 2015;42(6):1197-1211.

11. Hamerman JA, Pottle J, Ni M, He Y, Zhang ZY, Buckner JH. Negative regulation of TLR signaling in myeloid cells - implications for autoimmune diseases. Immunol Rev. 2016;269(1):212-227.

12. Blander JM. The many ways tissue phagocytes respond to dying cells. Immunol Rev. 2017;277(1):158-173. 
13. Guilliams M, Mildner A, Yona S. Developmental and functional heterogeneity of monocytes. Immunity. 2018;49(4):595-613. 14. Watowich SS, Liu YJ. Mechanisms regulating dendritic cell specification and development. Immunol Rev. 2010;238(1):76-92.

15. Li HS, et al. The signal transducers STAT5 and STAT3 control expression of Id2 and E2-2 during dendritic cell development. Blood. 2012;120(22):4363-4373.

16. Anderson KL, Perkin H, Surh CD, Venturini S, Maki RA, Torbett BE. Transcription factor PU.1 is necessary for development of thymic and myeloid progenitor-derived dendritic cells. J Immunol. 2000;164(4):1855-1861.

17. Guerriero A, Langmuir PB, Spain LM, Scott EW. PU.1 is required for myeloid-derived but not lymphoid-derived dendritic cells. Blood. 2000;95(3):879-885.

18. Bakri Y, et al. Balance of MafB and PU.1 specifies alternative macrophage or dendritic cell fate. Blood. 2005;105(7):2707-2716

19. Sarrazin S, et al. MafB restricts M-CSF-dependent myeloid commitment divisions of hematopoietic stem cells. Cell. 2009;138(2):300-313

20. Goudot C, et al. Aryl hydrocarbon receptor controls monocyte differentiation into dendritic cells versus macrophages. Immunity. 2017;47(3):582-596.e6.

21. Lowes MA, Suárez-Fariñas M, Krueger JG. Immunology of psoriasis. Annu Rev Immunol. 2014;32:227-255.

22. Boehncke WH, Schön MP. Psoriasis. Lancet. 2015;386(9997):983-994.

23. Xu M, et al. An Interleukin-25-mediated autoregulatory circuit in keratinocytes plays a pivotal role in psoriatic skin inflammation. Immunity. 2018;48(4):787-798.e4.

24. Hawkes JE, Yan BY, Chan TC, Krueger JG. Discovery of the IL-23/IL-17 signaling pathway and the treatment of psoriasis J Immunol. 2018;201(6):1605-1613.

25. Wohn C, et al. Langerin(neg) conventional dendritic cells produce IL-23 to drive psoriatic plaque formation in mice. Proc Natl Acad Sci U S A. 2013;110(26):10723-10728.

26. Singh TP, et al. Monocyte-derived inflammatory Langerhans cells and dermal dendritic cells mediate psoriasis-like inflammation. Nat Commun. 2016;7:13581.

27. Bartel DP. MicroRNAs: genomics, biogenesis, mechanism, and function. Cell. 2004;116(2):281-297.

28. Mehta A, Baltimore D. MicroRNAs as regulatory elements in immune system logic. Nat Rev Immunol. 2016;16(5):279-294.

29. Tsokos GC, Lo MS, Costa Reis P, Sullivan KE. New insights into the immunopathogenesis of systemic lupus erythematosus. Nat Rev Rheumatol. 2016;12(12):716-730.

30. Zhou H, et al. MicroRNA-223 regulates the differentiation and function of intestinal dendritic cells and macrophages by targeting C/EBP $\beta$. Cell Rep. 2015;13(6):1149-1160.

31. Pan W, et al. MicroRNA-21 and microRNA-148a contribute to DNA hypomethylation in lupus CD4 ${ }^{+} \mathrm{T}$ cells by directly and indirectly targeting DNA methyltransferase 1. J Immunol. 2010;184(12):6773-6781.

32. Qingjuan L, et al. miR-148a-3p overexpression contributes to glomerular cell proliferation by targeting PTEN in lupus nephritis. Am J Physiol Cell Physiol. 2016;310(6):C470-C478.

33. Gonzalez-Martin A, et al. The microRNA miR-148a functions as a critical regulator of B cell tolerance and autoimmunity. Nat Immunol. 2016;17(4):433-440.

34. Liu X, et al. MicroRNA-148/152 impair innate response and antigen presentation of TLR-triggered dendritic cells by targeting CaMKII . J Immunol. 2010;185(12):7244-7251.

35. Goedeke L, et al. MicroRNA-148a regulates LDL receptor and ABCA1 expression to control circulating lipoprotein levels. Nat Med. 2015;21(11):1280-1289.

36. Suzuki HI, Young RA, Sharp PA. Super-enhancer-mediated RNA processing revealed by integrative microRNA network analysis. Cell. 2017;168(6):1000-1014.e15.

37. Zhu Y, et al. miR-148a inhibits colitis and colitis-associated tumorigenesis in mice. Cell Death Differ. 2017;24(12):2199-2209.

38. Hashimi ST, Fulcher JA, Chang MH, Gov L, Wang S, Lee B. MicroRNA profiling identifies miR-34a and miR-21 and their target genes JAG1 and WNT1 in the coordinate regulation of dendritic cell differentiation. Blood. 2009;114(2):404-414.

39. Lu C, et al. miR-221 and miR-155 regulate human dendritic cell development, apoptosis, and IL-12 production through targeting of p27kip1, KPC1, and SOCS-1. Blood. 2011;117(16):4293-4303.

40. Ceppi M, et al. MicroRNA-155 modulates the interleukin-1 signaling pathway in activated human monocyte-derived dendritic cells. Proc Natl Acad Sci U S A. 2009;106(8):2735-2740.

41. Dunand-Sauthier I, et al. Silencing of c-Fos expression by microRNA-155 is critical for dendritic cell maturation and function. Blood. 2011;117(17):4490-4500.

42. Guinea-Viniegra J, et al. Targeting miR-21 to treat psoriasis. Sci Transl Med. 2014;6(225):225re1.

43. Xu L, Leng H, Shi X, Ji J, Fu J, Leng H. MiR-155 promotes cell proliferation and inhibits apoptosis by PTEN signaling pathway in the psoriasis. Biomed Pharmacother. 2017;90:524-530.

44. Wu R, et al. MicroRNA-210 overexpression promotes psoriasis-like inflammation by inducing Th1 and Th17 cell differentiation. J Clin Invest. 2018;128(6):2551-2568.

45. Cai Y, et al. Pivotal role of dermal IL-17-producing $\gamma \delta$ T cells in skin inflammation. Immunity. 2011;35(4):596-610.

46. Lai Y, et al. The antimicrobial protein REG3A regulates keratinocyte proliferation and differentiation after skin injury. Immunity. 2012;37(1):74-84.

47. Agarwal V, Bell GW, Nam JW, Bartel DP. Predicting effective microRNA target sites in mammalian mRNAs. Elife. $2015 ; 4$. 